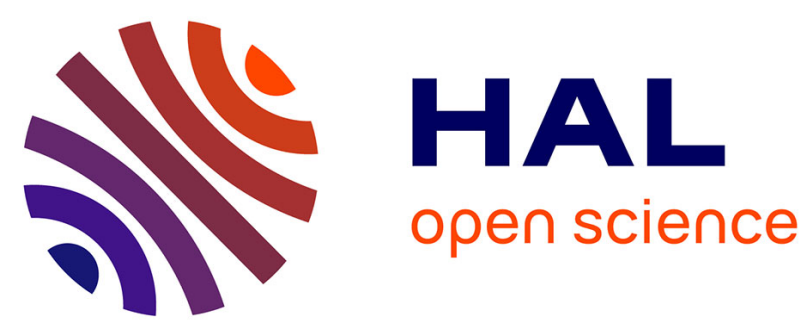

\title{
Dynamics, radiation and overall energy budget of earthquake rupture with coseismic off-fault damage
}

Kurama Okubo, Harsha S Bhat, Esteban Rougier, Samson Marty, Alexandre Schubnel, Zhou Lei, Earl Knight, Yann Klinger

\section{- To cite this version:}

Kurama Okubo, Harsha S Bhat, Esteban Rougier, Samson Marty, Alexandre Schubnel, et al.. Dynamics, radiation and overall energy budget of earthquake rupture with coseismic off-fault damage. Journal of Geophysical Research : Solid Earth, 2019, 124, pp.11771 - 11801. 10.1029/2019JB017304 . hal-02324458

\section{HAL Id: hal-02324458 \\ https://hal.science/hal-02324458}

Submitted on 26 Mar 2021

HAL is a multi-disciplinary open access archive for the deposit and dissemination of scientific research documents, whether they are published or not. The documents may come from teaching and research institutions in France or abroad, or from public or private research centers.
L'archive ouverte pluridisciplinaire HAL, est destinée au dépôt et à la diffusion de documents scientifiques de niveau recherche, publiés ou non, émanant des établissements d'enseignement et de recherche français ou étrangers, des laboratoires publics ou privés. 


\section{JGR Solid Earth}

\author{
RESEARCH ARTICLE \\ 10.1029/2019JB017304 \\ Key Points: \\ - Earthquake ruptures dynamically \\ activate coseismic off-fault damage, \\ whose feedback plays an important \\ role in rupture dynamics \\ - We show the mechanism of \\ dynamically activated off-fault \\ fractures and its effect on rupture \\ velocity and enhanced \\ high-frequency radiation \\ - The contribution of off-fault \\ damage to the overall energy budget \\ associated with earthquakes is \\ nonnegligible even at depth
}

Correspondence to:

K. Okubo,

okubo@geologie.ens.fr

Citation:

Okubo, K., Bhat, H. S., Rougier, E., Marty, S., Schubnel, A., Lei, Z., et al. (2019). Dynamics, radiation, and overall energy budget of earthquake rupture with coseismic off-fault damage. Journal of Geophysical Research: Solid Earth,124, 11,771-11,801. https://doi.org/10.1029/ 2019JB017304

Received 4 JAN 2019

Accepted 2 AUG 2019

Accepted article online 6 AUG 2019

Published online 28 NOV 2019

(C)2019. American Geophysical Union. All Rights Reserved.

\section{Dynamics, Radiation, and Overall Energy Budget of Earthquake Rupture With Coseismic Off-Fault Damage}

\author{
Kurama Okubo ${ }^{1,2,3}(\mathbb{D})$, Harsha S. Bhat ${ }^{2}(\mathbb{D})$, Esteban Rougier ${ }^{4}(\mathbb{D})$ Samson Marty $^{2}(\mathbb{D})$, \\ Alexandre Schubnel ${ }^{2}(\mathbb{D})$, Zhou Lei ${ }^{4} \mathbb{( D}$, Earl E. Knight ${ }^{4}(\mathbb{D})$, and Yann Klinger ${ }^{1}(\mathbb{D}$ \\ ${ }^{1}$ Institut de Physique du Globe de Paris, Sorbonne Paris Cité, Université Paris Diderot, UMR 7154 CNRS, Paris, France, \\ ${ }^{2}$ Laboratoire de Géologie, École Normale Supérieure/CNRS UMR 8538, PSL Research University, Paris, France, \\ ${ }^{3}$ Department of Earth and Planetary Sciences, Harvard University, Cambridge, MA, USA, ${ }^{4}$ EES-17-Earth and \\ Environmental Sciences Division, Los Alamos National Laboratory, Los Alamos, NM, USA
}

\begin{abstract}
Earthquake ruptures dynamically activate coseismic off-fault damage around fault cores. Systematic field observation efforts have shown the distribution of off-fault damage around main faults, while numerical modeling using elastic-plastic off-fault material models have demonstrated the evolution of coseismic off-fault damage during earthquake ruptures. Laboratory-scale microearthquake experiments have pointed out the enhanced high-frequency radiation due to the coseismic off-fault damage. However, the detailed off-fault fracturing mechanisms, subsequent radiation, and its contribution to the overall energy budget remain to be fully understood because of limitations of current observational techniques and model formulations. Here, we constructed a new physics-based dynamic earthquake rupture modeling framework, based on the combined finite-discrete element method, to investigate the fundamental mechanisms of coseismic off-fault damage, and its effect on the rupture dynamics, the radiation and the overall energy budget. We conducted a 2-D systematic case study with depth and showed the mechanisms of dynamic activation of the coseismic off-fault damage. We found the decrease in rupture velocity and the enhanced high-frequency radiation in near field due to the coseismic off-fault damage. We then evaluated the overall energy budget, which shows a significant contribution of the coseismic off-fault damage to the overall energy budget even at depth, where the damage zone width becomes narrower. The present numerical framework for the dynamic earthquake rupture modeling thus provides new insights into earthquake rupture dynamics with the coseismic off-fault damage.
\end{abstract}

Plain Language Summary The medium surrounding fault cores can be damaged due to stress concentration caused by the dynamic earthquake ruptures propagating on the faults, which is called coseismic off-fault damage. Systematic field observation efforts have shown the distribution of off-fault damage around main faults, while numerical modeling has demonstrated the evolution of off-fault damage during earthquake ruptures. Laboratory-scale microearthquake experiments have pointed out the enhanced high-frequency radiation due to the off-fault damage. However, the detailed off-fault fracturing mechanisms, subsequent seismic wave radiation and its contribution to the overall energy budget remain to be fully understood. Here, we constructed a new physics-based dynamic earthquake rupture modeling framework to investigate the fundamental mechanisms of coseismic off-fault damage and its effect on the rupture dynamics, the radiation, and the overall energy budget. We found the enhanced high-frequency radiation in near field due to the coseismic off-fault damage. We then evaluated the overall energy budget, which shows a significant contribution of the coseismic off-fault damage to the overall energy budget even at depth. The present numerical framework for the dynamic earthquake rupture modeling thus provides the insight into the earthquake rupture dynamics with the coseismic off-fault damage.

\section{Introduction}

Coseismic off-fault damage is recognized as a key factor towards understanding the physics of dynamic earthquake ruptures and the associated overall energy budget. Sibson (1977) conceptually proposed a formulation for the overall energy budget of dynamic earthquake ruptures; a part of the energy released from accumulated strain energy by interseismic deformation is converted to seismic wave radiation, whereas the 
rest is expended in inelastic deformation processes within fault zone. Wallace and Morris (1986) then characterized the structure of fault zones from the observation of deep mines in North America, where fault cores are surrounded by fractured rock. Chester and Logan (1986) and Chester et al. (1993) also proposed similar fault zone structures based on field observations of San Gabriel and Punchbowl faults in Southern California.

Figure 1 illustrates the schematic of a hierarchical fault structure across length scales ranging from regional fault systems to microfractures. The geometrical complexity of fault system is usually discussed in kilometric scale (Figures 1a and 1b). However, when focusing on a part of a fault system, smaller-scale fracture networks are observed around faults after the earthquake rupture propagates on the main faults (Figure 1c). These mesoscopic off-fault fractures also have an effect on the displacement field around the faults (Cappa et al., 2014; Manighetti et al., 2004). Eventually, Figure 1d shows the fault zone structure involving microscopic fractures around the fault core. Field measurements of the microfracture density as a function of distance in fault-normal direction have been conducted in order to understand the spatial distribution and geometric characteristics of the off-fault damage zones (Faulkner et al., 2011; Mitchell \& Faulkner, 2009; Savage \& Brodsky, 2011; Shipton \& Cowie, 2001). Mitchell and Faulkner (2012) showed that the microfracture density is significantly higher close to the fault and exponentially decreases with distance from the fault core, evidencing the presence of coseismic off-fault damage in microscale (Figures 1e and 1f). Since all these geometrical complexities of fractures in a wide range of length scale play a role in the faulting process, the modeling of coseismic off-fault damage is crucial to better understand the rupture dynamics, the radiation, and the overall energy budget associated with earthquakes.

Numerous studies have been performed via theoretical approaches, experiments, and numerical modeling to evaluate the effect of coseismic off-fault damage on the earthquake ruptures. Poliakov et al. (2002) and Rice et al. (2005) showed the potential failure area around rupture front with steady-state cracks and pulses based on theoretical formulations. Marty et al. (2019) performed laboratory experiments of laboscale dynamic ruptures with sawcut rock specimens. They found enhanced high-frequency radiation in acoustic recordings during stick-slip events, though to be caused by the coseismic off-fault damage, which is of great interest for understanding the high-frequency components in near-field ground motion (Castro \& Ben-Zion, 2013; Hanks, 1982).

The numerical modeling of coseismic off-fault damage has been also conducted to demonstrate the evolution of the off-fault damage activated by dynamic earthquake ruptures and its effect on the rupture dynamics (Andrews, 2005; Ando \& Yamashita, 2007; Ben-Zion \& Shi, 2005; Bhat et al., 2012; Dalguer et al., 2003; Dunham et al., 2011a; Gabriel et al., 2013; Ma \& Andrews, 2010; Templeton \& Rice, 2008; Thomas \& Bhat, 2018; Yamashita, 2000; Viesca et al., 2008). However, up to now, the state-of-the-art numerical techniques used for earthquake rupture modeling were not able to describe detailed off-fault fracturing processes as actual tensile and shear (Mode I and Mode II) fractures mainly due to limitations of computation and model formulations. Hence, the role of coseismic off-fault damage activated by the dynamic earthquake ruptures remains to be fully understood. Therefore, our aim in this study is to model explicitly the activation of off-fault fracture networks by dynamic earthquake ruptures to evaluate the effect of coseismic off-fault damage on the rupture dynamics, the radiation, and the overall energy budget.

We used the combined finite-discrete element method (FDEM) to model the dynamic earthquake rupture with the coseismic off-fault damage. It allows for the activation of both off-fault tensile and shear fractures based on prescribed cohesion and friction laws so that we can quantify the effect of coseismic off-fault damage on the rupture dynamics, the radiation, and the overall energy budget.

We first demonstrate, for a canonical problem, 2-D dynamic earthquake rupture modeling with coseismic off-fault damage. We then show the mechanisms of secondary off-fault fractures, and its effect on the rupture velocity and the radiation. Eventually, we calculate the evolution of energy components associated with the dynamic earthquake rupture to investigate the overall energy budget.

\section{Dynamic Earthquake Rupture Modeling With Coseismic Off-Fault Damage}

We performed dynamic earthquake rupture modeling on a planar strike-slip fault under plane strain conditions, surrounded by an initially intact rock, allowing for the activation of off-fault fractures. Figure 2 a shows 


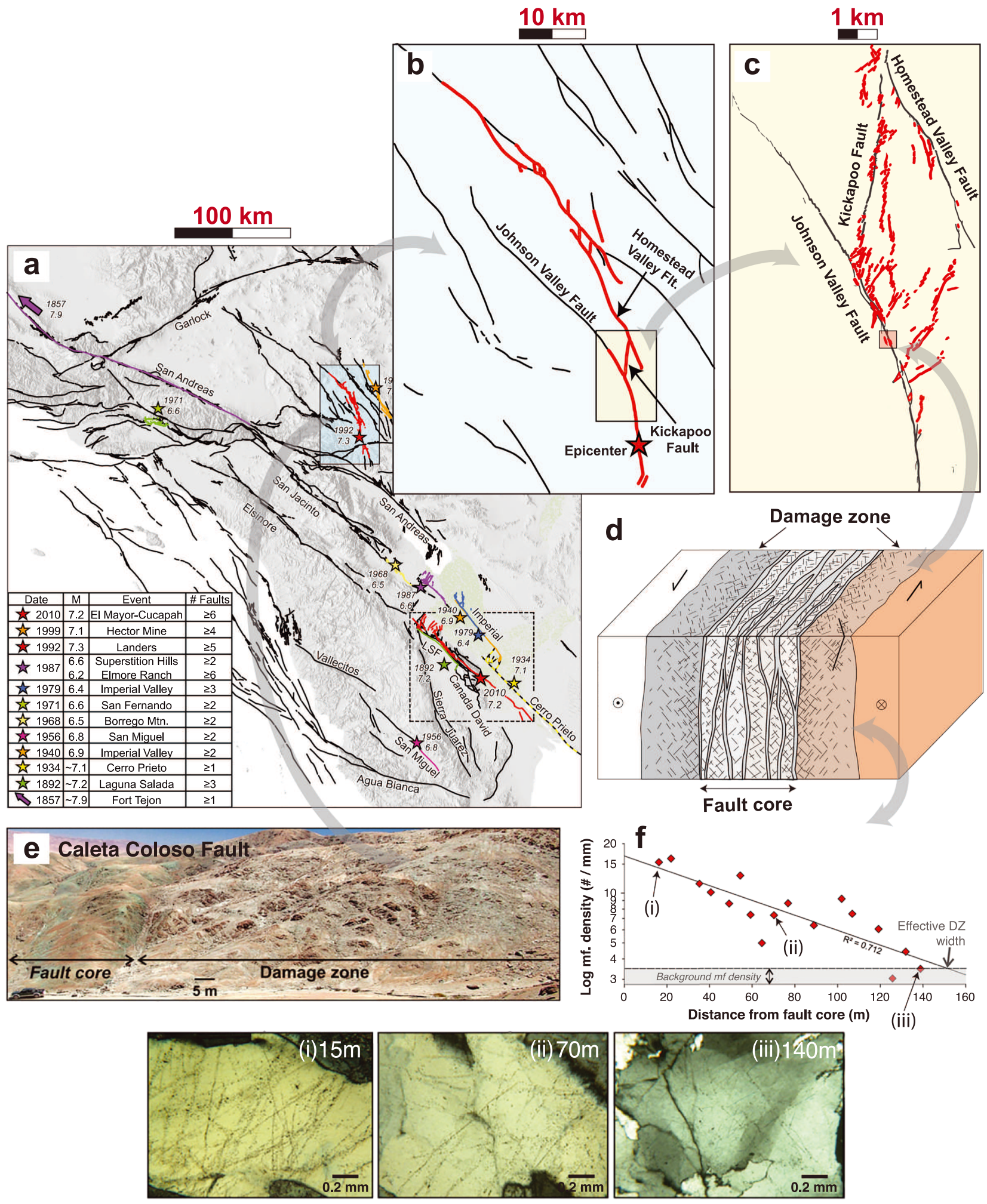

Figure 1. Hierarchical structure of fault systems over a wide range of length scales. (a) Fault map of Southern California (Fletcher et al., 2014). Black lines indicate the fault traces. Stars and colored lines indicate the epicenters and the rupture traces of historic earthquake events, respectively. (b) Fault map and the rupture traces (in red) associated with the 1992 Landers earthquake (modified from Sowers et al., 1994). (c) Smaller-scale off-fault fracture network (Sowers et al., 1994). (d) Schematic of fault zone structure, showing a fault core surrounded by damage zones (Mitchell \& Faulkner, 2009). (e, f) Fault damage zone of Caleta Coloso fault, the variation in microfracture (mf.) density within the damage zone as a function of distance from fault core and optical microscopic images of microfractures at different distances from the fault core. (Mitchell \& Faulkner, 2012). 


\section{a}
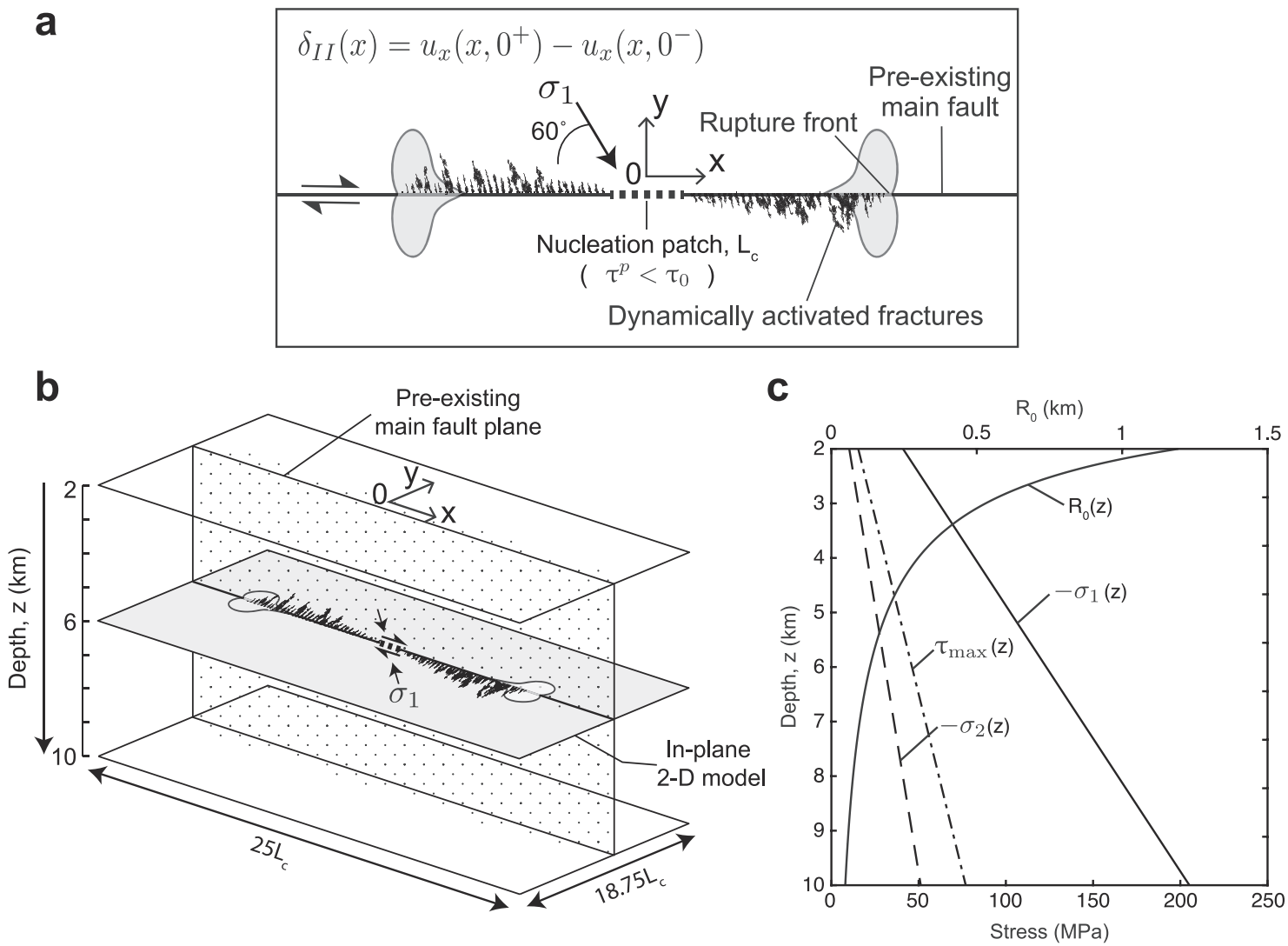

Figure 2. Model description for the case study with depth. (a) The 2-D strike-slip fault for dynamic rupture modeling with coseismic off-fault damage. The preexisting fault is defined as an interface without cohesion. The orientation of maximum compressional principal stress $\sigma_{1}$ is fixed at $60^{\circ}$ from the main fault. The slip on the fault $\delta_{I I}$ is defined as the relative displacement. (b) Schematic of case study with depth. (c) The evolution of initial stress state and quasi-static process zone size $R_{0}(z)$ with depth. $-\sigma_{1}(z),-\sigma_{2}(z), \tau_{\max }(z)$ indicate maximum principal stress, minimum principal stress and maximum shear traction, respectively.

the model description for the 2-D dynamic earthquake rupture modeling. The rupture is artificially nucleated from the nucleation patch, where the peak friction is lower than the initial shear traction on the main fault. The size of nucleation patch $L_{c}$ is determined by the critical crack length (Palmer \& Rice, 1973). Then it propagates bilaterally on the main fault, dynamically activating off-fault fractures. The $x$ axis is along the fault-parallel direction, while the $y$ axis is along the fault-normal direction. Figure $2 \mathrm{~b}$ shows the schematic of case study with depth. We performed a set of 2-D dynamic earthquake rupture modeling to investigate the evolution of coseismic off-fault damage and its effect with depth. The $z$ axis is thus along depth. We conducted 2-D simulations at every $1 \mathrm{~km}$ from $z=2$ - to $10-\mathrm{km}$ depth with corresponding initial stress state as shown in Figure 2c. We assume lithostatic condition with depth so that the confining pressure linearly increases with depth. The quasi-static process zone size $R_{0}$ (see equation (A16)) decreases with depth when the fracture energy on the main fault $G_{I I C}^{f}$ is kept constant with depth (Figure 2c). Note that the case study does not address 3-D effects (e.g., free surface) as we model the dynamic ruptures assuming 2-D plane strain conditions.

For the sake of fair comparison between different depths, the model parameters are nondimensionalized by scaling factors. $R_{0}(\mathrm{~m})$ and shear wave velocity $c_{s}(\mathrm{~m} / \mathrm{s})$ are used to scale the length $(\mathrm{m})$ and the time (s) by $R_{0}$ and $R_{0} / c_{s}$, respectively. Subsequently, other variables are also nondimensionalized by the combination of those two scaling factors. Since the density of medium does not change during simulations, the nondimensionalization of mass is not necessary in our problem.

The methodology of FDEM is described in Appendix A. More details of the numerical framework to model dynamic earthquake rupture with FDEM can be found in Okubo (2018). The parameters used for the case study with depth are summarized in Table A1. We used FDEM-based software tool, Hybrid Optimization Software Suite-Educational Version (HOSSedu) for the dynamic earthquake rupture modeling (Knight et al., 


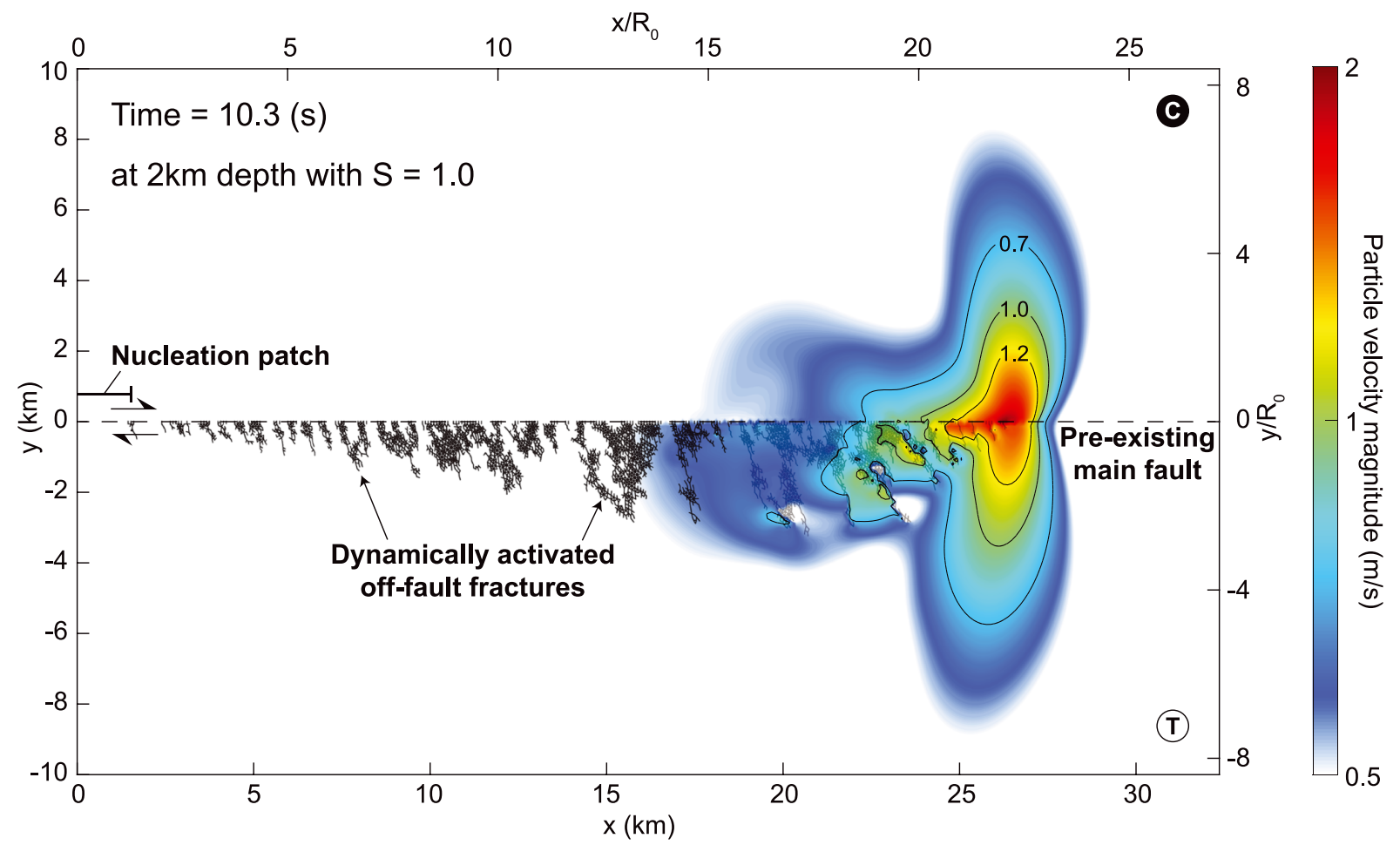

Figure 3. Snapshot of the dynamic earthquake rupture with coseismic off-fault damage. We plot only the right part $(x>0)$ as the result is symmetrical with respect to the origin. The initial stress state and the strength of material correspond to $2-\mathrm{km}$ depth with $S=1.0$. Color contour indicates the particle velocity magnitude in log scale. Dotted line indicates the main fault, and the solid lines indicate the off-fault fractures. The bottom and left axes show the physical length scale, while the top and right axes show the nondimensionalized length, scaled by $R_{0}$. "C" and " $T$ " at right corners indicate compressional and extensional side of the main fault, respectively.

2015). Before modeling dynamic earthquake ruptures with coseismic off-fault damage, we conducted the cross validation of the FDEM using purely elastic medium to assess the achievable accuracy of earthquake rupture modeling. The results are summarized in Appendix B.

Figure 3 shows a snapshot of dynamic earthquake rupture with dynamically activated off-fault fractures, where particle velocity field and the fracture traces around the main fault are superimposed. The seismic ratio $S$ is equal to 1.0 (see equation (A3)), which results in the sub-Rayleigh rupture during the simulation with the coseismic off-fault damage. The off-fault fractures are plotted when the traction applied on the potential failure plane (i.e., boundary of meshes) reaches the cohesive strength and the cohesion starts weakening. Bottom and left axes indicate the fault-parallel and fault-normal distance in physical length scale, while top and right axes indicate the nondimensionalized length scale.

The off-fault fractures are initiated around the rupture tip, and then it forms an intricate fracture network as the main rupture propagates on the main fault. The particle velocity field is significantly perturbed due to the coseismic off-fault damage. The extensional side of the main fault is mostly damaged, which is supported by the theoretical analysis of potential failure area (Poliakov et al., 2002; Rice et al., 2005) and other simulations (e.g., Andrews, 2005). The off-fault fractures form an intricate network by means of fracture coalescence, composed of tensile, shear, and mixed mode fractures. We later discuss this off-fault fracturing process for a case with a relatively steep angle between the maximum compressive principal stress $\sigma_{1}$ and the fault $\left(\psi=60^{\circ}\right)$ and its effect on the radiation in near field and the overall energy budget.

Figure 4 shows a set of snapshots for the supershear case with $S=0.7$. The rupture is nucleated and propagates with sub-Rayleigh speed in the earlier phase. Then a daughter crack is born ahead of the rupture front at $T=4.7 \mathrm{~s}$, which then transitions to supershear rupture. During the rupture transition from sub-Rayleigh to supershear, characteristic damage pattern appears; there is a gap of coseismic off-fault damage around the transition phase (around $x=12 \mathrm{~km}$ in Figure 4). This characteristic damage gap has been also pointed out by Templeton and Rice (2008) and Thomas and Bhat (2018). This can be explained by the Lorentz contraction 


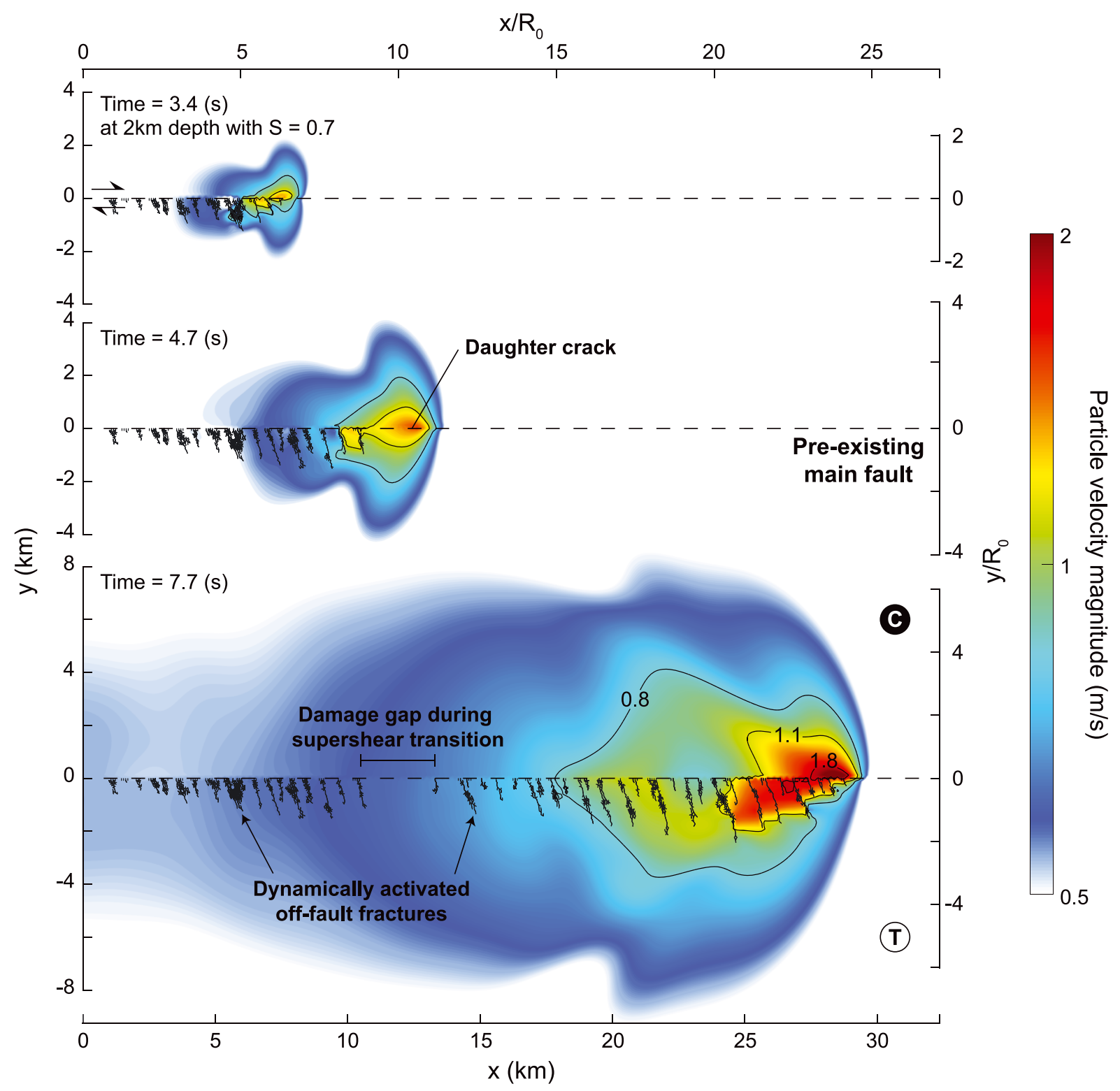

Figure 4. Snapshots of supershear rupture at $2-\mathrm{km}$ depth with $S=0.7$. Color contour and lines indicate the same as Figure 3 . The rupture velocity is sub-Rayleigh until $T=3.4 \mathrm{~s}$ (top), then a daughter crack is born ahead of the sub-Rayleigh rupture front at $T=4.7 \mathrm{~s}$ (middle), which then transitions to supershear (bottom).

of the dynamic process zone size $R_{f}\left(v_{r}\right)$ (see Appendix A4). The dynamic process zone size asymptotically shrinks at the limiting speed of the rupture. Since the damage zone size is approximately of the same order as the process zone size, the damage gap appears during the supershear transition. Then it resumes the off-fault fracturing after the initiation of supershear rupture. We need to further explore the transition of stress concentration associated with the dynamic rupture during supershear transition in order to better explain the mechanism of damage gap.

In the present study, we examined the cases with $S=1.0$ and 0.7 to simulate sub-Rayleigh and supershear ruptures with coseismic off-fault damage, respectively. However, the examined $S$ ratios are relatively low as both $S=1.0$ and 0.7 lead to supershear transition without the off-fault damage as shown in Figure 10 . The analysis of the effect of coseismic off-fault damage with larger $S(>1.5)$ remains to be done as the dominant wing cracks are activated from the edges of the nucleation patch during the nucleation phase, which prevents the rupture nucleation and the propagation along the main fault. Therefore, to avoid the huge stress concentration at the edge of the nucleation patch, we need to improve the nucleation process. 

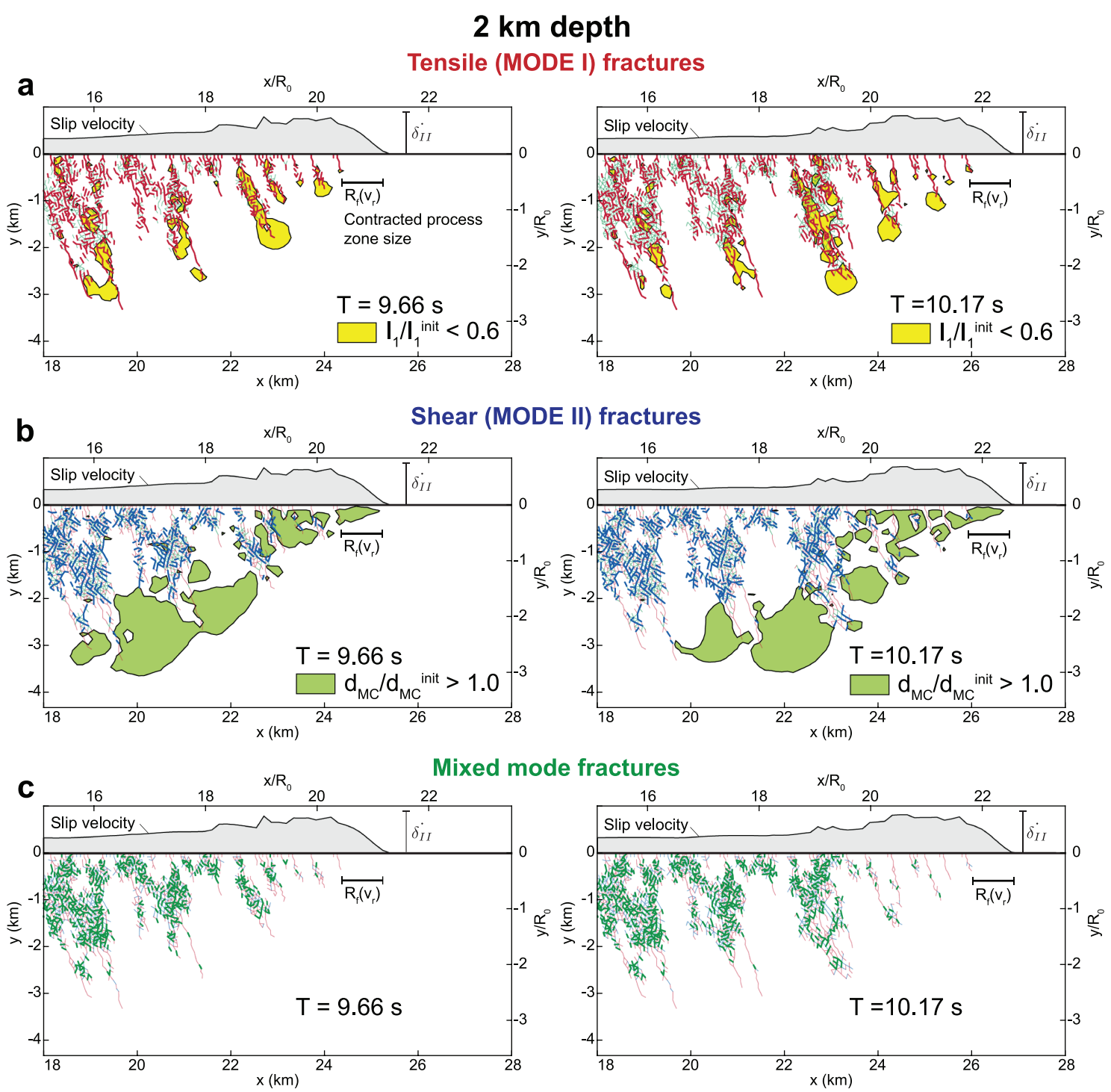

Figure 5. Off-fault fracturing process in tensile, shear and mixed mode with $S=1.0$ at 2-km depth. (a) Trace of tensile fractures at $T=9.66$ and $10.17 \mathrm{~s}$. Red heavy lines indicate the tensile fracture with damage type $D_{T} \geq 0.9$ (equation (A10)) and damage $D \geq 0.01$. Solid line on the top of the main fault indicates the slip velocity on the main fault. The filled area in yellow shows the potential failure area where the ratio of the first stress invariant to its initial value $I_{1}(t) / I_{1}^{\text {init }}$ is less than 0.6. The lighter lines in the fracture network indicate shear and mixed mode fractures. $R_{f}\left(v_{r}\right)$ shows the dynamic process zone size of the earthquake rupture on the main fault. (b) Trace of shear fractures. Blue heavy lines indicate the shear fracture with damage type $D_{T} \leq 0.1$ and damage $D \geq 0.01$. The filled area in green shows where the ratio of closeness to failure to its initial value $d_{\mathrm{MC}} / d_{\mathrm{MC}}^{\text {init }}>1.0$. $d_{\mathrm{MC}}^{\text {init }}$ is uniformly equal to 0.4 in the domain. (c) Trace of mixed mode fractures with $0.1<D_{T}<0.9$ and $D \geq 0.01$.

\section{Mechanism of Coseismic Off-Fault Damage}

We first investigate the fracturing process in off-fault medium activated by the dynamic rupture propagation. We aim to show how the off-fault fracture network evolves as the dynamic earthquake rupture propagates on the main fault. Figure 5 shows the traces of tensile (dilating), shear, and mixed mode fractures in the off-fault medium at two time steps replotted from Figure 3. To highlight the potential failure area, the first stress invariant normalized by its initial value $I_{1}(t) / I_{1}^{\text {init }}$ (equation (A17)) for tensile fractures and the normalized closeness to failure $d_{\mathrm{MC}} / d_{\mathrm{MC}}^{\text {init }}$ (equation (A14)) for shear fractures are, respectively, superimposed on the traces of secondary fractures (see section A5). Note that both regions do not assure the traction reaches the peak cohesion due to the threshold for plotting. Thus, the potential failure planes in the regions are not necessarily broken. 


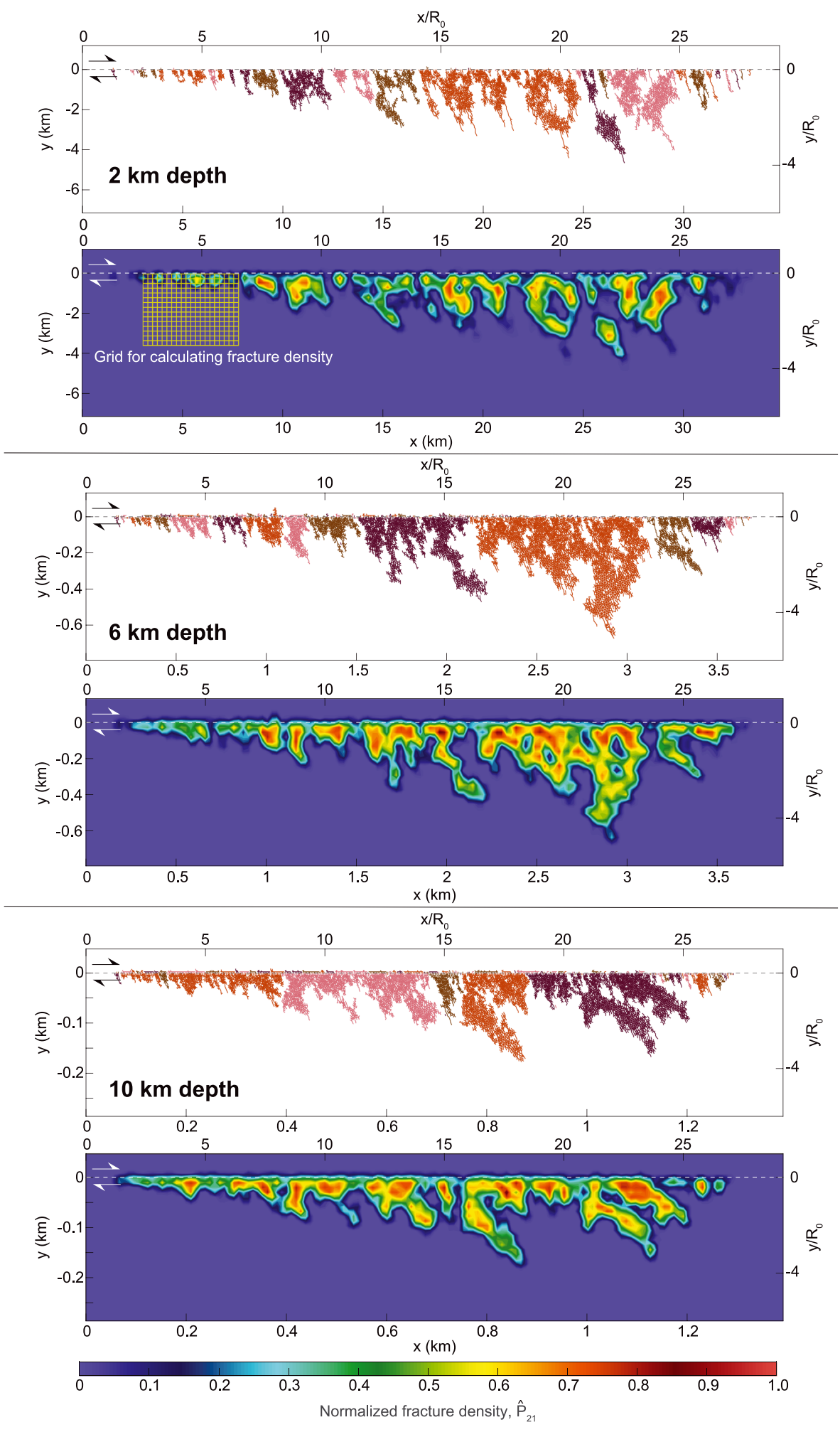

Figure 6. Off-fault fracture network and spatial distribution of fracture density with depth. The results are the final snapshot of simulations with $S=1.0$. An isolated fracture network, in which all small fractures connect with each other, is indicated by different colors. Color contour indicates the normalized fracture density $\hat{P}_{21}$. 

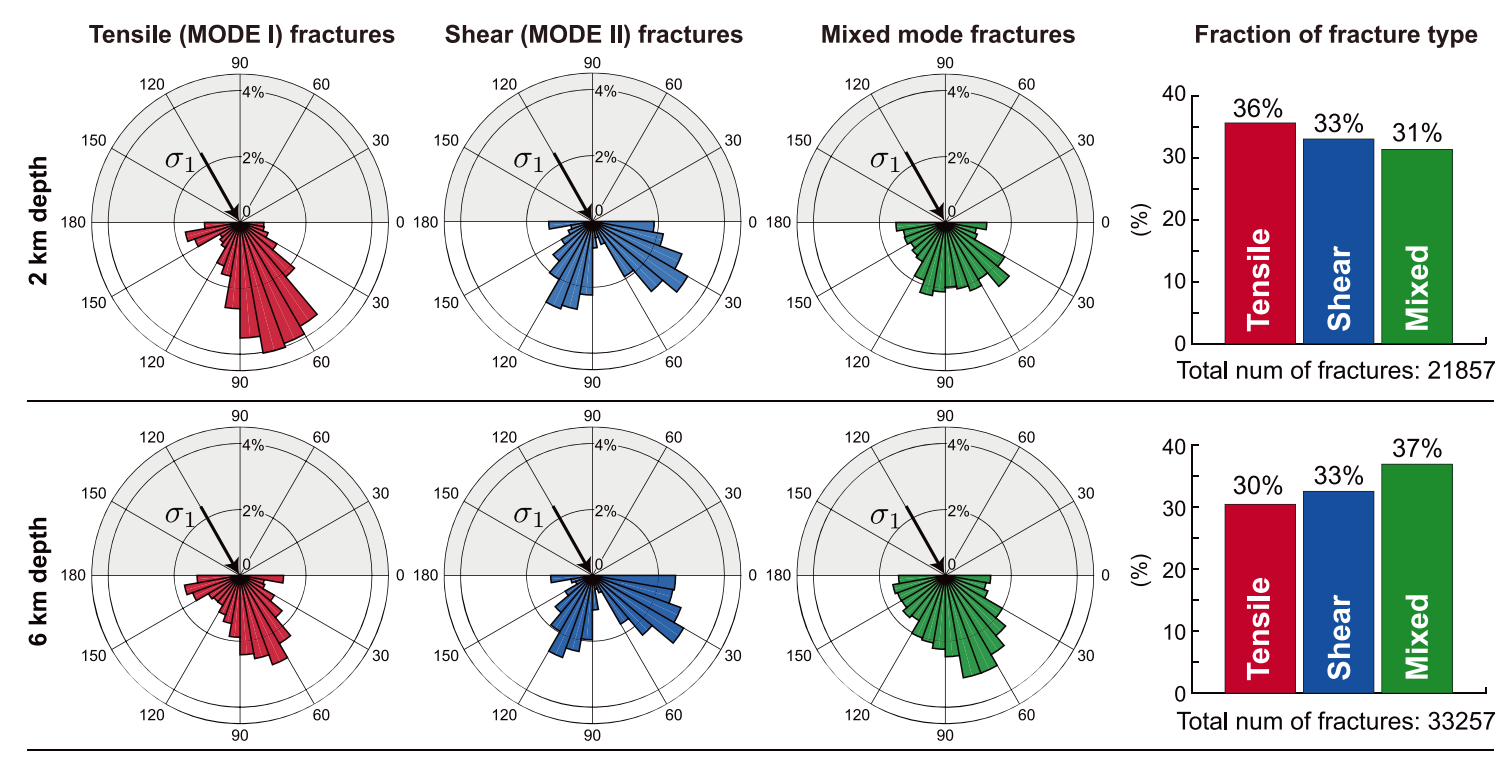

Total num of fractures: 21857
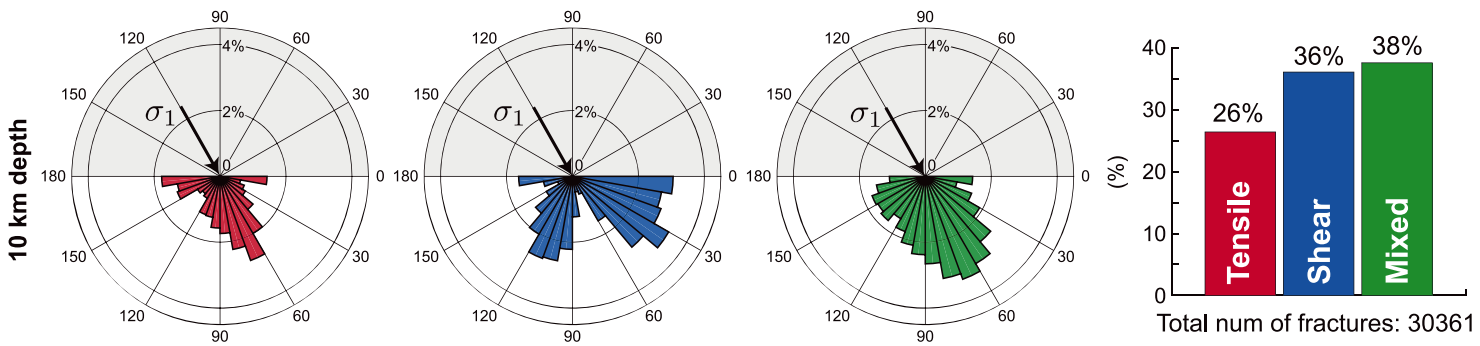

Figure 7. Rose diagram showing the orientation of secondarily activated fractures obtained from Figure 6 and the fraction of each type of fracture. Size of bars in rose diagram is normalized by the sum of all types of fracture. The arrow indicates the orientation of maximum compressive principal stress $\sigma_{1}$ on the main fault.

The intricate fracture network continues to evolve even after rupture front passes because stress concentrations still remain behind the rupture front due to the internal feedback from the off-fault fracture network itself. The stress concentration is then relaxed by the activation of new fractures in the off-fault medium. The tensile fractures are always initiated just behind the rupture front with a certain dominant orientation. This dominant orientation is experimentally and theoretically studied by Ngo et al. (2012) and has a reasonable correspondence with the orientation obtained from our analysis. It is also remarkable that the position of fracture initiation is always at the end of the dynamic process zone $R_{f}\left(v_{r}\right)$ (see section A4), which is also pointed out by Viesca et al. (2009).

We then examined the effect of depth to investigate the evolution of damage pattern, fracture density, and the damage zone width with depth. Figure 6 shows the traces of off-fault fracture network and the spatial distribution of fracture density at 2-, 6-, and 10-km depths. Isolated fracture networks, in which all fractures coalesce with each other, are separately plotted with different colors. The dimensions are scaled by $R_{0}$ so that the size of the fracture network is visually comparable. The number of isolated fracture network is more for the shallower case than the deeper case, implying the off-fault fracture network becomes more intricate and denser with depth. To evaluate the distribution of fracture density, we first imposed representative square grids around the fault as shown in Figure 6 at 2-km depth and calculated the normalized fracture density $\hat{P}_{21}$ in the each grid defined as

$$
\hat{P}_{21}=\frac{\text { length of fracture trace in a grid }}{\text { area of grid }} R_{0} .
$$

We carefully chose the grid size such that each grid has a reasonable number of fractures. In this analysis, the grid size is set to $0.2 R_{0}$, which is, on average, 3 times larger than the size of fractures. For the sake of comparison between different depths, the magnitude of $\hat{P}_{21}$ is normalized by its maximum value at $10-\mathrm{km}$ depth. We found that the fracture density globally increases with depth, though it does not monotonically 


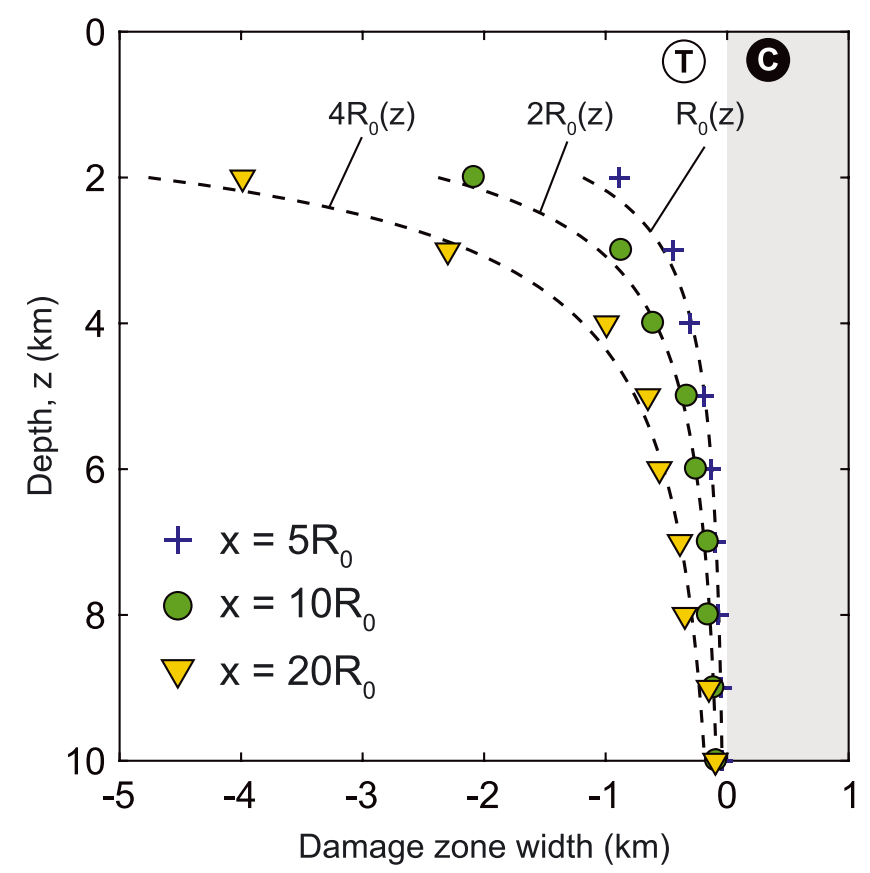

Figure 8. Evolution of damage zone width with depth. Markers indicate the damage zone width obtained from the case study. Type of markers indicate the position on the main fault at which the damage zone width is evaluated. The dotted lines indicate the quasi-static process zone size scaled by constant factors. increase due to complicated internal feedback in the off-fault fracture network. The increase in the normalized fracture density with depth enhances the contribution of coseismic off-fault damage to the overall energy budget, as discussed in section 6 . Note that the pulverization in the vicinity of main fault is not modeled because of limitations in the size of the potential failure planes. It would be resolved by incorporating constitutive damage models (Bhat et al., 2012).

Figure 7 shows the rose diagram showing the orientation of off-fault fractures. The size of bars in the rose diagram is normalized by the sum of all types of fracture. It has a dominant orientation for tensile fractures, which corresponds to the orientation of $\sigma_{1}$. The shear fractures also have two dominant orientations, which correspond to the conjugate failure planes inferred from the Mohr-Coulomb failure criterion. There is no dominant orientation for mixed mode fractures. The fraction of the each type of fracture shows that the population is fairly balanced, whereas the fraction of tensile fracture decreases with depth because more intricate fracture network is formed at depth.

Figure 8 shows the evolution of the damage zone width with depth. The damage zone is inferred from the envelope of secondary fracture network at scaled rupture lengths $x=5 R_{0}, 10 R_{0}$, and $20 R_{0}$ to compare at the same stage of dynamic ruptures with depth and to investigate representative damage zone width associated with the in situ confining pressure. Since there are few off-fault fractures being activated on the compressional side, we only plot the damage zone width on the extensional side. The damage zone width follows, up to a constant factor, the quasi-static process zone size. Hence, the damage zone width decreases with depth, which contributes to the formation of flower-like structure, with fracture connectivity increasing with depth. The evolution of damage zone width with depth has a first-order agreement with observations (e.g., Cochran et al., 2009).

We have here demonstrated the fracturing process of off-fault medium activated by the dynamic earthquake rupture with depth. The dynamic activation of off-fault fracture network has an effect on the rupture dynamics and causes additional radiation, which effects high-frequency components in near-field ground motion discussed in the upcoming section. We also examined the mesh dependency of the fracturing process because the potential failure planes are restricted to the element boundary, which is discussed in Appendix C.

\section{Rupture Velocity}

We next focus on the rupture velocity on the main fault. Figure 9 shows the evolution of slip velocity on the main fault with four cases; $S=1.0$ or 0.7 at $2-\mathrm{km}$ depth, each of which with or without off-fault damage. For the cases without off-fault damage, the activation of secondary fracture is suppressed by extremely high cohesion for both tensile and shear fractures. Here, we plot the contour of slip velocity in space and time. In Figure 9a, there is a clear transition from sub-Rayleigh to supershear around $x / R_{0}=20$, which is also shown in the inset. However, when the coseismic off-fault damage is taken into account, the supershear transition is not observed during the simulation as shown in Figure 9b. Hence, the secondary fractures can arrest, or delay, supershear transition under certain stress conditions. This can be explained by the increase in critical slip distance due to the coseismic off-fault damage. The supershear transition length $L^{\text {trans }}$ can be estimated from Andrews' result (Andrews, 1985; Xia et al., 2004) as follows:

$$
L^{\text {trans }}=\frac{1}{9.8\left(S_{\text {crit }}-S\right)^{3}} \frac{1+v}{\pi} \frac{\tau^{p}-\tau^{r}}{\left(\tau-\tau^{r}\right)^{2}} \mu D_{c}
$$

where $S_{\text {crit }}$ is the threshold for the supershear transition $\left(S_{\text {crit }}=1.77\right.$ for 2-D), $v$ is Poisson's ratio, $\tau_{p}, \tau_{r}$, and $\tau$ are peak friction (equation (A6)), residual friction (equation (A7)), and shear traction on the fault, respectively, $\mu$ is shear modulus, and $D_{c}$ is critical slip distance for friction (equation (A13)). $D_{c}$ is initially 

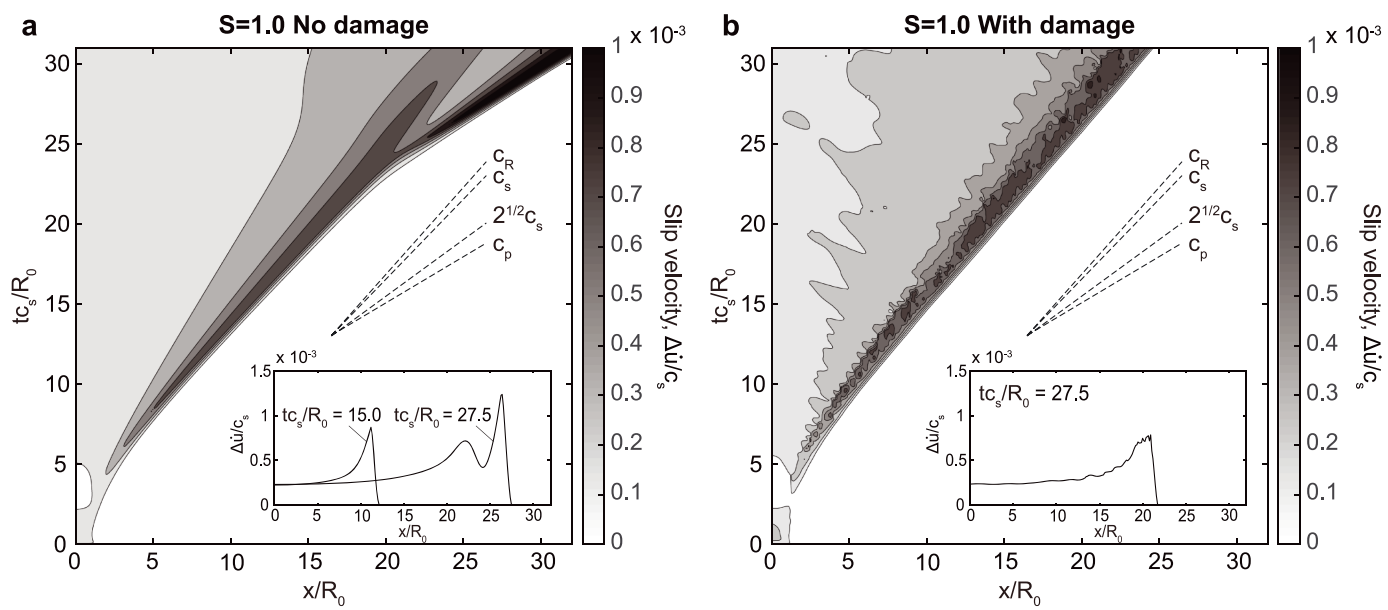

C

d
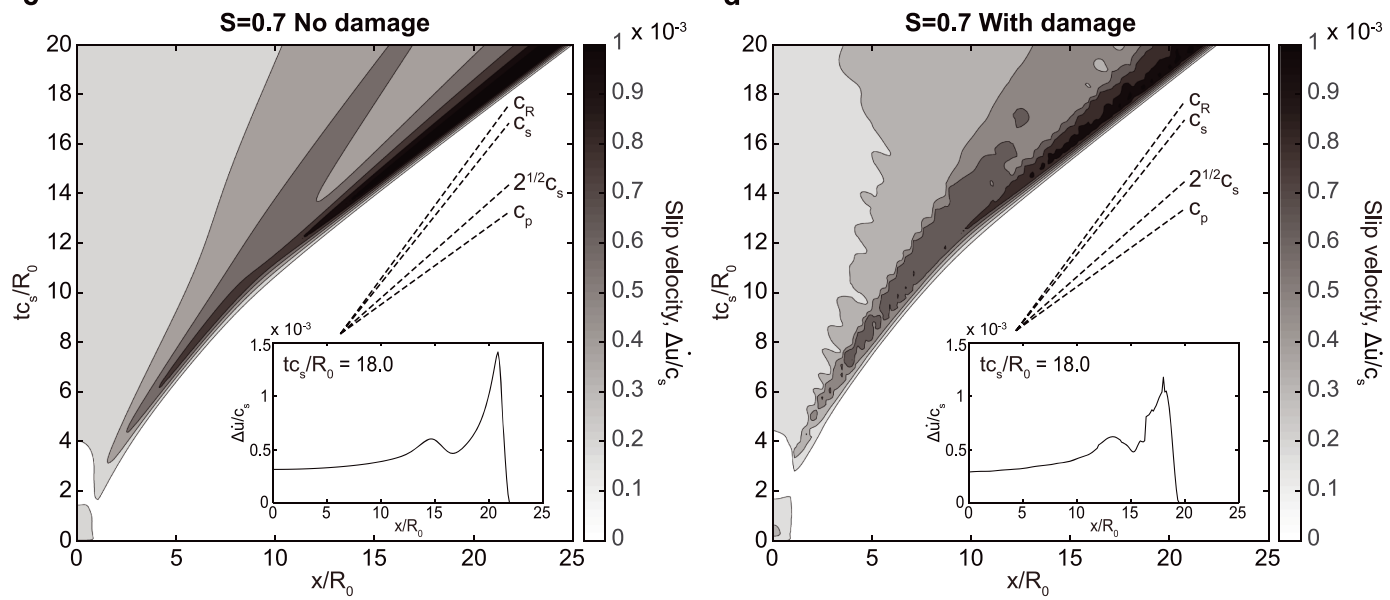

Figure 9. The evolution of slip velocity in time and space at $2-\mathrm{km}$ depth. There are four cases: (a) $S=1.0$ with no damage in the off-fault medium, (b) $S=1.0$ with damage, (c) $S=0.7$ with no damage, and (d) $S=0.7$ with damage. For the cases without damage, we set extremely high cohesion for both tensile and shear fractures so that the off-fault medium behaves as a purely elastic material. Color contour indicates the slip velocity. Dotted lines indicate the reference of the slope corresponding to each wave velocity. Insets show the distribution of slip velocity on the main fault at a certain time.

uniform on the main fault. However, the effective critical slip distance $D_{c}^{\text {eff }}$, which takes into account the energy dissipation in the off-fault medium due to the coseismic off-fault damage, increases with the rupture length as discussed in later section(section 6.1). Therefore, $L^{\text {trans }}$ also increases due to the coseismic off-fault damage as it is proportional to $D_{c}$.

Figures 9c and 9d show the cases with $S=0.7$, where the rupture transitions to supershear for both cases with and without off-fault damage because of the large contrast of the initial shear traction to the normal traction on the main fault. The time of supershear transition is delayed with off-fault damage due to the decrease of rupture velocity, whereas the difference of transition length is still obscure with these results. The two insets in the figures show the clear difference in the peak of slip velocity. In addition, the rupture arrival is delayed by the coseismic off-fault damage, implying the decrease in rupture velocity.

The rupture velocity is calculated from first arrival times along the main fault. Figure 10 shows the evolution of rupture velocity in time. We take the time derivatives of first arrival time in discretized space along the main fault to calculate the representative rupture velocity at a certain position. Since it is difficult to capture the exact time when rupture velocity jumps to supershear, where the curve of first arrival time has a kink and is nondifferentiable, the error caused by the smoothing of the rupture velocity is taken into account as shown by the error bars in Figure 10. Therefore, the markers in the forbidden zone $c_{R}<v_{R}<c_{S}$ do not conclusively indicate that the rupture velocity is between them due to the uncertainty. 


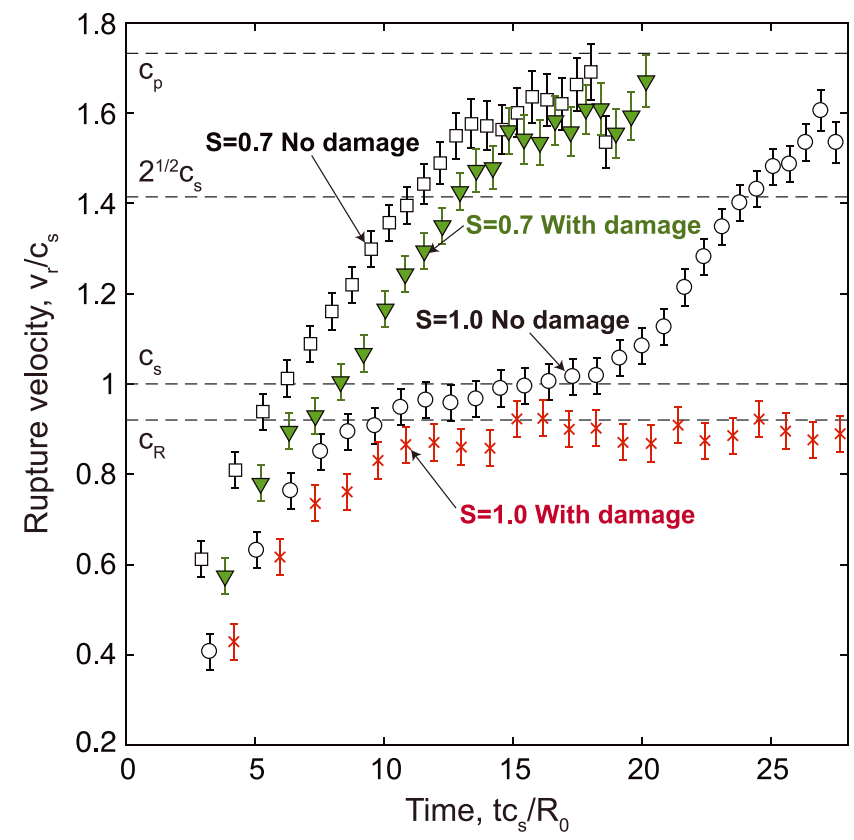

Figure 10. Rupture velocity inferred from Figure 9. Due to inherent discretization errors, it is difficult to precisely capture the jump of rupture velocity from sub-Rayleigh to supershear. The error is estimated from the difference between the slope of $c_{R}$ and $c_{S}$, the grid spacing and the sampling rate of slip velocity.

Regardless of the uncertainty, the comparison between the cases with and without off-fault damage shows the effect of coseismic off-fault damage on the rupture velocity and the supershear transition. The rupture transitions to supershear for both cases with $S=0.7$, whereas the rate of increase in rupture velocity is lower for the case with the off-fault damage. However, the supershear transition is suppressed due to the coseismic off-fault damage with $S=1.0$. Further parametric study would narrow down the criteria of supershear transition and would provide supershear transition length.

\section{High-Frequency Radiation in Near Field}

The origin of high-frequency radiation has been studied over decades (e.g., Castro \& Ben-Zion, 2013; Dunham et al., 2011b; Hanks, 1982; Hanks \& McGuire, 1981; Madariaga, 1977; Marty et al., 2019; Ohnaka et al., 1987; Passelègue et al., 2016). There are multiple factors that effect the high-frequency radiation, such as sudden nucleation and arrest of rupture, complex fault geometry, roughness of the fault surface, and the nonlinear response in subsurface sedimentary rock. In this study, we propose that the coseismic off-fault damage could also contribute to high-frequency radiation. When secondary fractures are activated in the off-fault medium, they behave as secondary sources of radiation, which contribute to the enhancement of high-frequency components in the near-field ground motion. The off-fault fracture network also causes scattering due to structural heterogeneities.

Figure 11a shows the waveforms of the fault-normal acceleration at $x=12.4 R_{0}, 2-\mathrm{km}$ depth with $S=1.0$. The amplitude is compressed to highlight the signals arising from the coseismic off-fault damage. The theoretical $P$ and $S$ wave arrival time and the rupture arrival time at $x=12.4 R_{0}$ are indicated in Figure 11a. After the $P$ and $S$ wave arrival, there is a well-aligned signal around $6 \mathrm{~s}$, which is caused by the stress perturbation around the rupture front. Significant high-frequency spikes then arise after the main rupture arrival, which are caused by the secondary off-fault fracturing, instead of the regular near-field radiation from the main rupture. These spikes are observed up to $2 R_{0}$ from the fault, corresponding to the damage zone width at this rupture length. Figure $11 \mathrm{~b}$ shows the spectrogram of the near-field ground acceleration $\left(y=-0.5 R_{0}\right)$. The spikes are observed at $t=8.7$ and $9.9 \mathrm{~s}$ even after the passage of rupture on the main fault due to the secondary fracturing activated by the internal feedback of off-fault fracture network.

We then investigate the spatial distribution of the high-frequency radiation with depth using the critical frequency $f^{\text {crit }}$, where the amplitude spectrum decays from the mean level of low-frequency band. Figure 12 


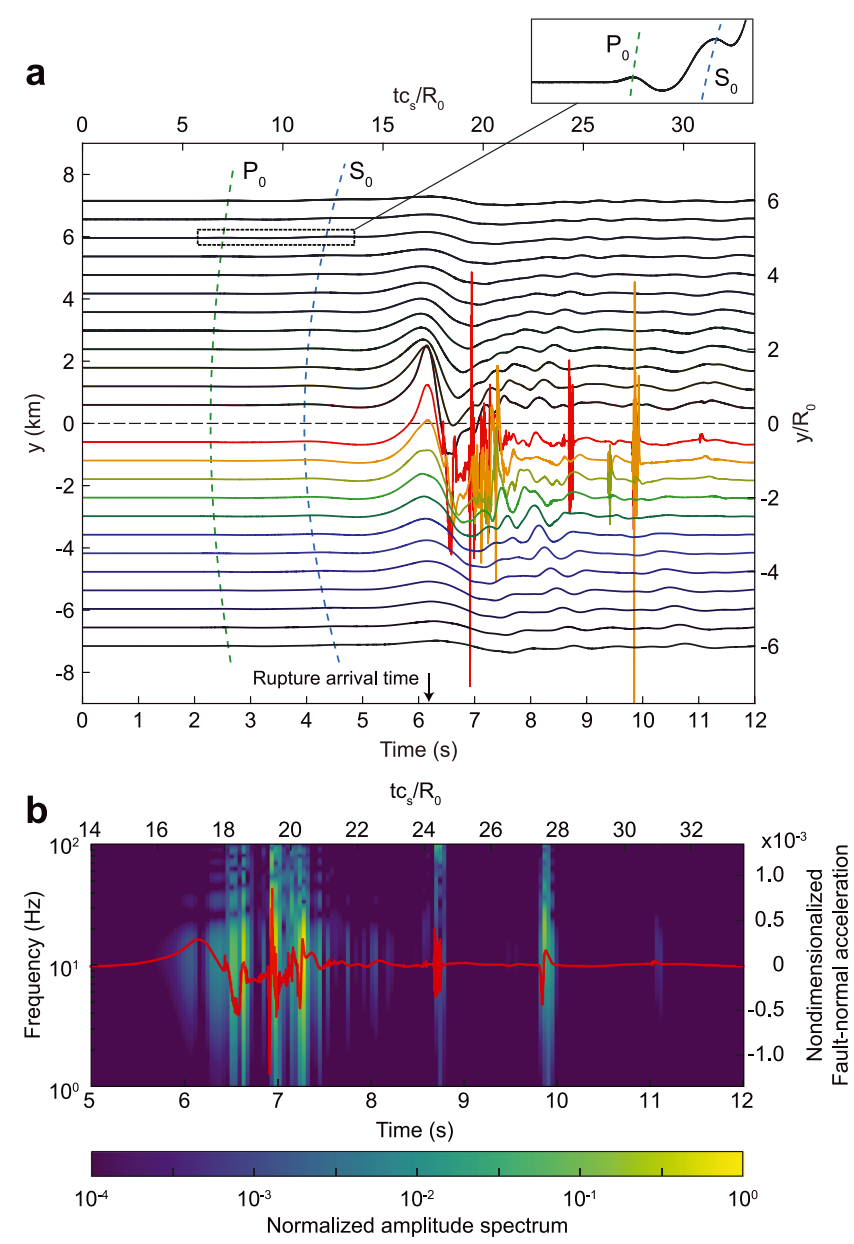

Figure 11. (a) Fault-normal acceleration at $x=12.4 R_{0}, 2-\mathrm{km}$ depth with $S=1.0$. Line colors of waveform indicate the fault-normal distance. Dotted lines indicate the theoretical $P$ and $S$ wave arrival time. The inset shows magnified signals, where the $P$ and $S$ arrival can be found. The rupture arrival time at the location of stations $\left(x=12.4 R_{0}\right)$ is $6.2 \mathrm{~s}$, indicated by the arrow. (b) Spectrogram of the near-field ground acceleration $\left(y=-0.5 R_{0}\right)$. The amplitude spectrum is normalized by its maximum value over the time.

shows the spatial distribution of $f^{\text {erit }}$ and the near- and far-fault spectra. Note that the far-fault does not mean far-field ground motion, where the near-field and intermediate-field terms are negligible in a point source model. The near-fault amplitude spectrum in the right column of Figure 12 is evaluated within the damage zone, which is not intended for direct comparison to the observations as it is recognized that there might be technical issues related with the deployment of instruments so close to the fault.

The signal time window starts from the first arrival time at the location to the end of simulation. We applied a band-pass filter of $0.1-100 \mathrm{~Hz}$ and a Tukey window. The spectra for the case without off-fault damage are superimposed at $2-\mathrm{km}$ depth for the comparison. The results show significant high-frequency radiation caused by the secondary fractures, which propagates even outward from the damage zone. Therefore, although the high-frequency radiation is quickly attenuated due to geometric spreading, the coseismic off-fault damage is clearly one of the factors that affects the high-frequency radiation in the near field.

The enhanced high-frequency radiation associated with dynamic ruptures is also hinted in experiments. Marty et al. (2019) conducted systematic stick-slip experiments with sawcut westerly granites under servo-controlled triaxial loading with the confining pressure $\sigma_{3}$ ranging from 10 to $90 \mathrm{MPa}$ to investigate the enhanced high-frequency radiation in acoustic recordings of the stick-slip events. The acoustic sensors are externally located on the surface of specimen, which record the motion of the normal component to the surface. The representative Fourier spectra are obtained by taking an average of 13 acoustic sensors on the 

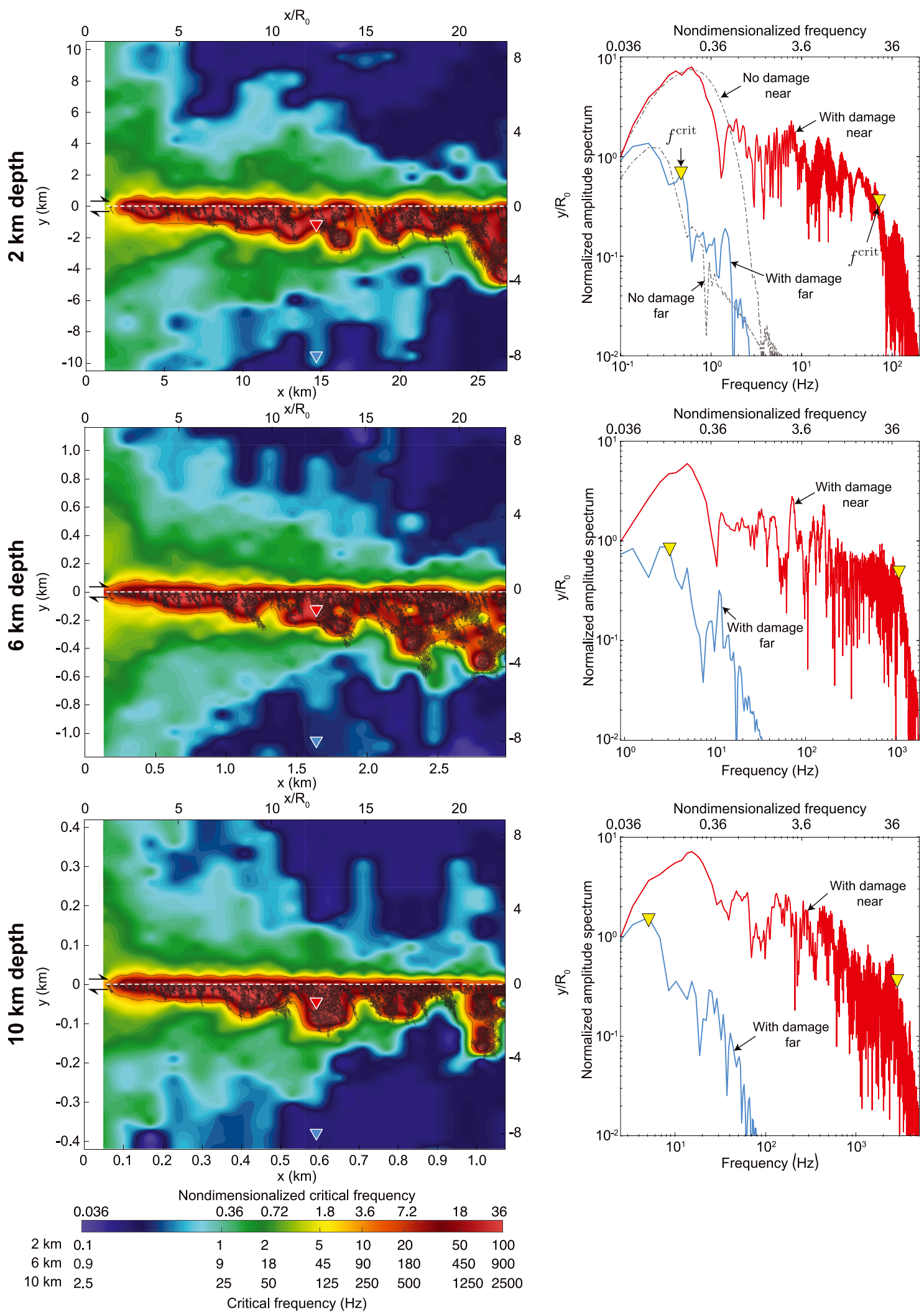

Figure 12. Spatial distribution of critical frequency and spectra of fault-normal acceleration with depth. Color contour shows the critical frequency. The off-fault fractures are superimposed. Inverted triangles indicate the locations of the stations. The spectra for the cases without off-fault damage at 2-km depth are indicated by the dashed lines in gray. 


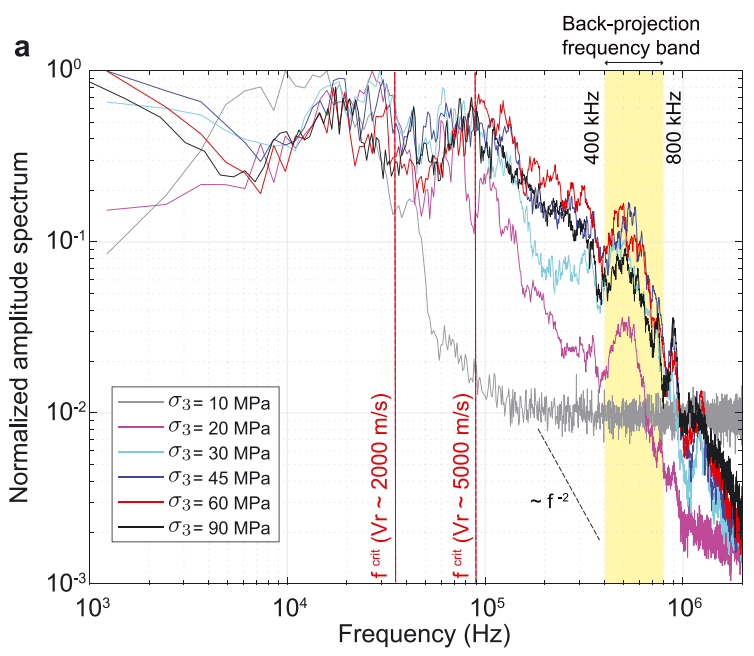

b
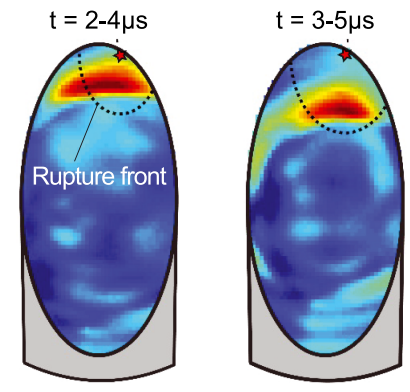

Location of rupture nucleation

Figure 13. Enhanced high-frequency radiation and back-projection analysis in laboratory experiments (Marty et al., 2019). (a) Fourier spectra with different confining pressures. Red dashed lines indicate the theoretical critical frequencies at $v_{r}=2,000 \mathrm{~m} / \mathrm{s}$ and $v_{r}=5,000 \mathrm{~m} / \mathrm{s}$. Highlighted box indicates the frequency band used for the back-projection analysis. (b) Snapshots of back-projection results (band-pass filtered from 400 to $800 \mathrm{kHz}$ ) with $\sigma_{3}=90 \mathrm{MPa}$ at $t=2-4,3-5$, and 4-6 $\mu$ s relative to the onset of rupture. Red star indicates the nucleation position. Dashed line indicates the theoretical rupture front. Color contour shows the normalized coherency function, which indicates the most likely location of the origin of signals within the frequency band.

specimen to get rid of directivity effect. Further information of these experiments can be found in Marty et al. (2019).

Figure 13a shows the Fourier spectra with different confining pressures. Each spectrum amplitude is normalized by its corresponding stress drop in order to compare the high-frequency content. The theoretical critical frequencies for $v_{r}=2,000 \mathrm{~m} / \mathrm{s}$ (for sub-Rayleigh) and $v_{r}=5,000 \mathrm{~m} / \mathrm{s}$ (for supershear) are indicated, implying the rupture transitions to supershear with $\sigma_{3} \geq 20 \mathrm{MPa}$. Certainly, one of the possible reasons for the enhanced high-frequency components is the supershear transition. However, there is also an enhanced frequency band from 400 to $800 \mathrm{kHz}$, which can be caused by the coseismic off-fault damage. Thus, they conducted back-projection analysis to investigate the spatiotemporal evolution of seismic energy release in this frequency band.

Figure $13 \mathrm{~b}$ shows snapshots of back-projection results for a certain stick-slip event with $\sigma_{3}=90 \mathrm{MPa}$. The color contour indicates the normalized coherency function, which shows the most likely location of the origin of the signal within the frequency band. The rupture is spontaneously nucleated at the edge of the sawcut surface and propagates downward. The theoretical rupture front is also superimposed on the fault surface. The results show that the high-frequency signals within this band originate just behind the rupture front, which can be caused by the coseismic off-fault damage. This can be compared to the off-fault fracturing process discussed in section 3. The experimental results thus highly suggest that the source of high-frequency radiation are the off-fault fractures that nucleate behind the main rupture front as shown in Figure 5. 
a
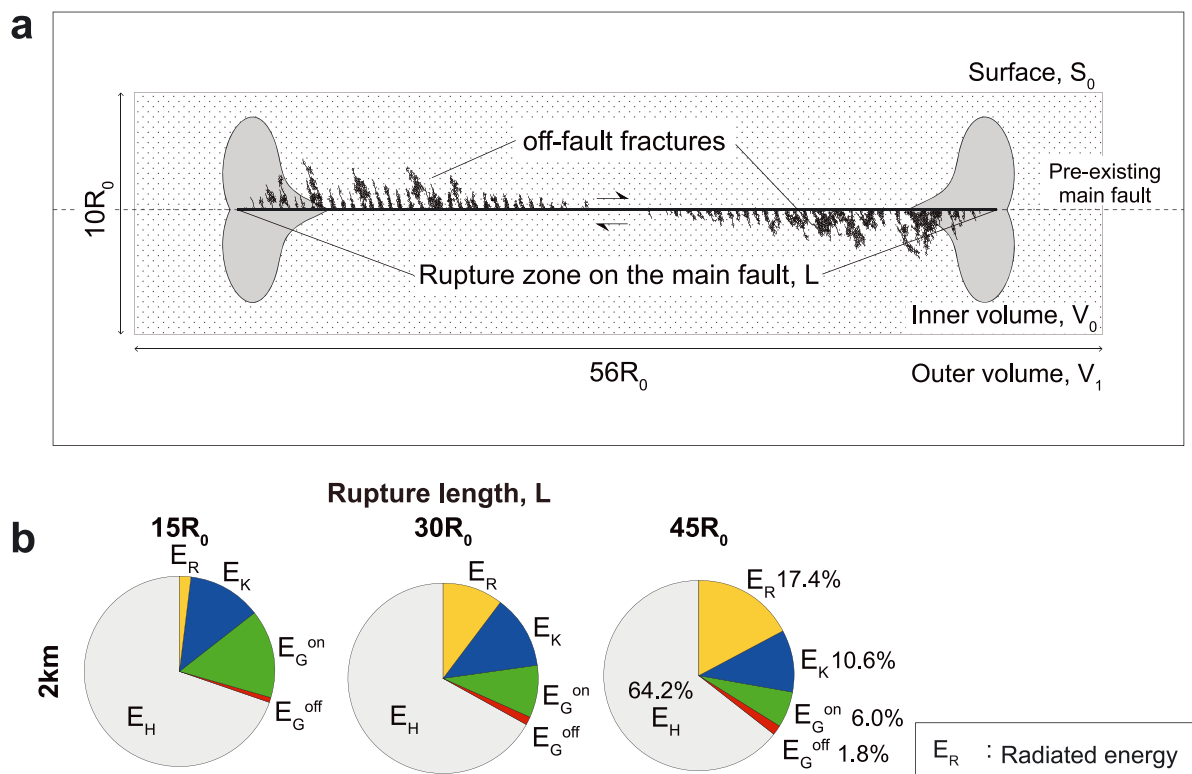

Rupture length, $\mathrm{L}$
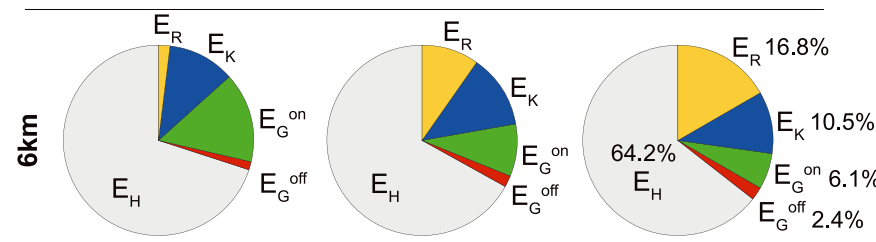
$\mathrm{E}_{\mathrm{R}} \quad$ : Radiated energy The work done by $\mathrm{V}_{0}$ to $\mathrm{V}_{1}$
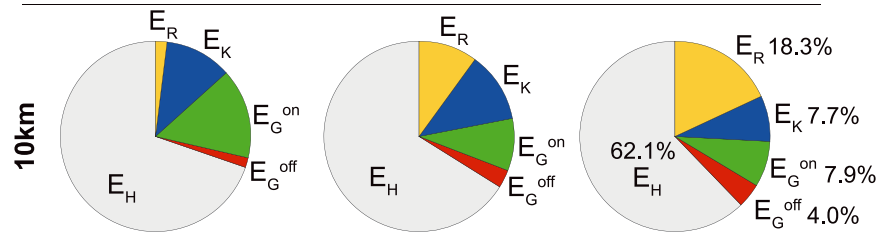

$\mathrm{E}_{\mathrm{K}}$ : Kinetic energy

$E_{G}{ }^{\text {on }: \text { Fracture energy }} \begin{aligned} & \text { on the main fault } \\ & \text { on }\end{aligned}$

$E_{\text {off }}$ Total fracture energy

$E_{H}$ : Heat energy

Figure 14. Schematic for overall energy budget calculation and the fraction of energy components. (a) Schematic for overall energy budget. The dotted area shows the inner volume $V_{0}$ with surface $S_{0}$, where the energy budget is evaluated. The inner volume is rectangular with unit thickness in our calculation. The size of the target area is arbitrarily chosen as $10 R_{0} \times 56 R_{0}$. (b) Fraction of energy components against $-\left(\Delta W+E_{S_{0}}^{0}\right)$ as a function of rupture length with depth. The results are for the case with $S=1.0$.

\section{Overall Energy Budget}

The overall energy budget of an earthquake event plays a key role in understanding the characteristics of the earthquake source, the change of potential energy, and the radiation. Here, we first describe the formulation of energy balance, which can be used to evaluate the overall energy budget associated with the dynamic earthquake rupture with coseismic off-fault damage. Although there are various approaches to derive the energy conservation law of earthquake ruptures (e.g., Fukuyama, 2005; Rivera \& Kanamori, 2005; Shi et al., 2008; Xu et al., 2012), we reidentify the energy components in a suitable form for the analysis of the overall energy budget within our numerical framework.

The overall energy budget is evaluated in an inner volume $V_{0}$ as shown in Figure $14 \mathrm{a}$, which encompasses the entire rupture zone and off-fault fractures. Then the energy components associated with the overall energy budget are written as follows:

- Elastic strain energy

$$
\Delta W=\int_{V_{0}}\left[\int_{0}^{\varepsilon_{i j}} \sigma_{i j} d \varepsilon_{i j}\right] \mathrm{d} V,
$$


where $\varepsilon_{i j}$ is strain tensor and $\sigma_{i j}$ is stress tensor. Note that the initial strain is defined to be zero, whereas the initial stress is nonzero. This configuration is commonly used in seismology, discussed in Aki \& Richards (2002, Box 8.5).

- Kinetic energy

$$
E_{K}=\int_{V_{0}} \frac{1}{2} \rho \dot{u}_{i} \dot{u}_{i} \mathrm{~d} V
$$

where $\rho$ is density and $\dot{u}_{i}$ is particle velocity.

- Radiated energy

$$
E_{R}(t)=-\int_{0}^{t} \mathrm{~d} t \int_{S_{0}}\left(T_{i}-T_{i}^{0}\right) \dot{u}_{i} \mathrm{~d} S
$$

where $S_{0}$ is the closed surface of $V_{0}, T_{i}$ is the traction on $S_{0}$, and $T_{i}^{0}=T_{i}(0)$. $E_{R}$ is essentially the work done by $V_{0}$ to outer volume $V_{1}$. Note that the canonical $E_{R}$ is determined at the end of earthquake event, where $E_{K}=0$ in $V_{0}$ (Kostrov, 1974), whereas $E_{R}$ defined by equation (5) is a function of time due to our model description with infinite fault length, where the rupture does not cease during simulation.

- Fracture energy on the main fault

$$
E_{G}^{\text {on }}=\int_{\Gamma_{\text {Main Fault }}}\left[\int_{\delta_{I I}^{f, e}}^{\min \left\{D_{c}^{\text {main }}, \delta_{I I}^{*}\right\}} T_{t}\left(\delta_{I I}\right)-\tau_{r} \mathrm{~d} \delta\right] \mathrm{d} S
$$

where $\Gamma^{\text {Main Fault }}$ is the surface of main fault, $\delta_{I I}^{f, e}$ is the critical slip for elastic loading of friction (see section A2), $D_{c}^{\text {main }}$ is critical slip distance of slip-weakening law on the main fault, $\delta_{I I}^{*}$ is slip at $t$ and $T_{t}$ is shear traction on the fault. Note that $E_{G}^{o n}$ is always positive during the slip-weakening of friction.

- Fracture energy associated with the off-fault damage

$$
E_{G}^{\text {off }}=\sum_{i}^{N} \int_{\Gamma_{i}^{\text {Offf-fault }}}\left[\int_{\delta_{I / I I}^{c, e}}^{\min \left\{\delta_{I / I I}^{c, c}, \delta_{I / I I}^{*}\right\}} C_{I / I I}\left(\delta_{I / I I}\right) \mathrm{d} \delta+\int_{\delta_{I I}^{f, e}}^{\min \left\{D_{c}^{\text {off }}, \delta_{I I}^{*}\right\}} T_{t}\left(\delta_{I I}\right)-\tau_{r} \mathrm{~d} \delta\right] \mathrm{d} S,
$$

where $\Gamma_{i}^{\text {Off-fault }}$ is the surface of off-fault fracture, $N$ is the number of off-fault fractures, $\delta_{I / I I}^{c, e}$ is the critical slip for elastic loading of tensile and shear cohesion, $\delta_{I / I I}^{c, c}$ is the maximum displacement for softening of tensile and shear cohesion, $D_{c}^{\text {off }}$ is critical slip distance of slip-weakening law in the off-fault medium, $\delta_{I / I I}^{*}$ is opening and shear displacement at $t$, and $C_{I / I}$ is the tensile and shear cohesion.

- Heat energy

$$
E_{H}=\int_{\Gamma^{\text {Main Fault }}+\Gamma_{i}^{\text {Off-fault }}}\left[\int_{\delta_{I I}^{f, e}}^{\delta_{I I}^{*}} \tau_{r} \mathrm{~d} \delta\right] \mathrm{d} S
$$

Using the energy components described above, the overall energy budget is written as

$$
E_{R}+E_{K}+E_{G}^{\mathrm{on}}+E_{G}^{\mathrm{off}}+E_{H}=-\left(\Delta W+E_{S_{0}}^{0}\right),
$$

where

$$
E_{S_{0}}^{0}=-\int_{0}^{t} \mathrm{~d} t \int_{S_{0}} T_{i}^{0} \dot{u}_{i} \mathrm{~d} S
$$

$E_{S_{0}}^{0}$ originates from the definition of radiated energy in equation (5), which does not appear in the conventional energy conservation law on earthquake (e.g., Rivera \& Kanamori, 2005) because of reasonable approximation processes to estimate the radiated energy. In this study, however, we define the overall energy budget with $E_{S_{0}}^{0}$ to rigorously estimate the contribution of each energy components to the overall energy budget. The detailed derivation of the overall energy budget can be found in Okubo (2018). 

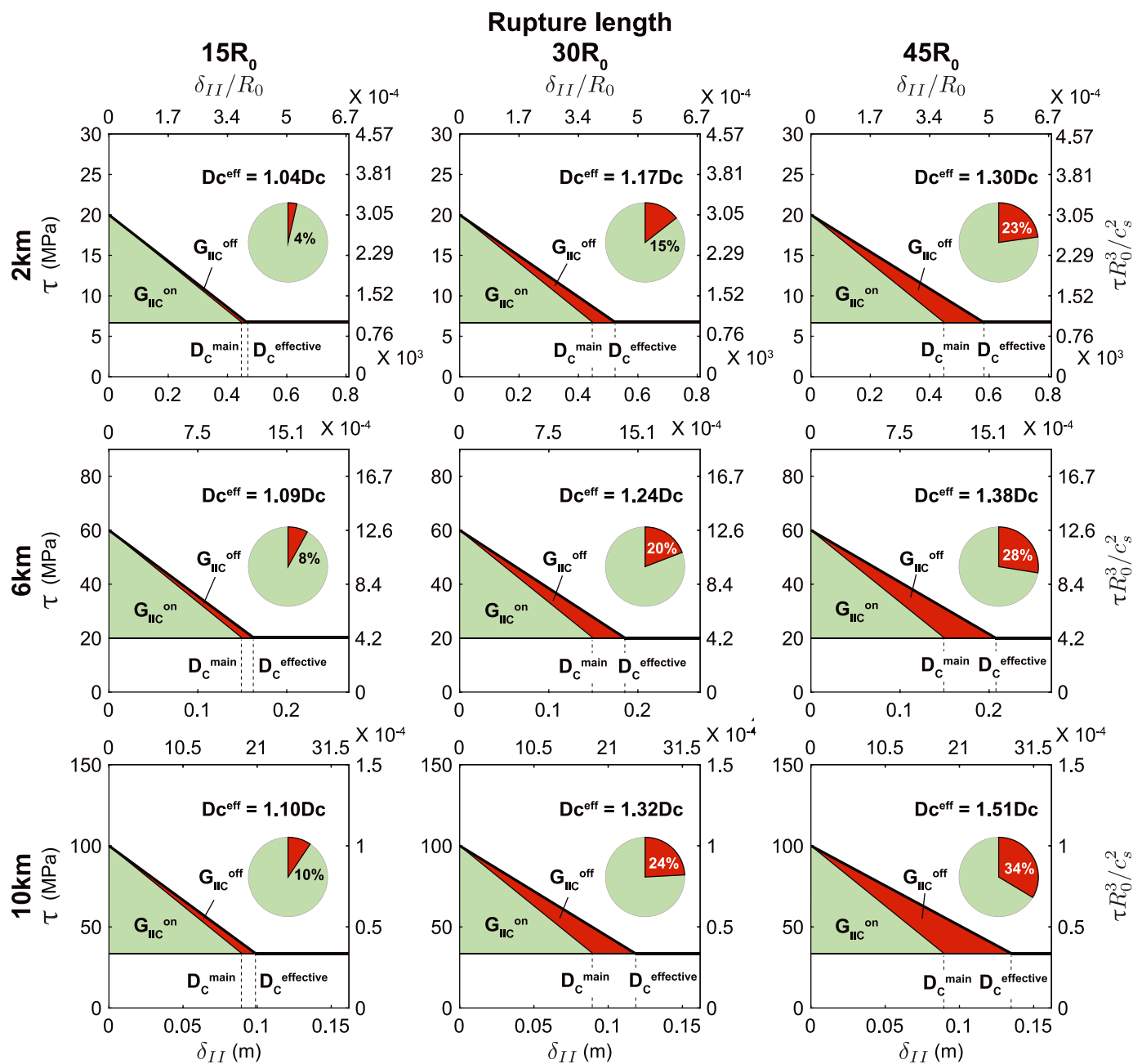

Figure 15. Effective $D_{c}$ derived from Figure 14b. $G_{I I C}^{\text {off }}$ indicates the total fracture energy dissipated due to the coseismic off-fault damage.The pie chart indicates the fraction of $E_{G}^{\text {off }}$ against $E_{G}^{\text {on }}$.

\subsection{Energy Dissipation in Off-Fault Medium}

Figure 14b shows the fraction of each energy components in the left side of equation (9) as a function of rupture length with depth. Each fraction is calculated against $-\left(\Delta W+E_{S_{0}}^{0}\right)$. Currently, $\Delta W, E_{S_{0}}^{0}, E_{R}, E_{K}$, and $E_{G}^{\text {on }}$ are directly calculated from the simulation, whereas $E_{G}^{\text {off }}$ and $E_{H}$ are indirectly evaluated with the assumption of average displacement on the off-fault fractures due to the current limitation of postprocessing. Note that the fracture energy $G_{I I C}^{f}$ is constant along the main fault. More details for the calculation of energy components can be found in Okubo (2018, section 3.4).

The fraction of fracture energy associated with the off-fault damage $E_{G}^{\text {off }}$ increases with the rupture length. Moreover, it also increases with depth even though damage zone width becomes narrower at depth.

To highlight the fraction of $E_{G}^{\text {off }}$ against $E_{G}^{\text {on }}$, we calculated the effective $D_{c}$ defined as

$$
D_{c}^{\mathrm{eff}}=D_{c}^{\mathrm{main}}+\frac{2 E_{G}^{\mathrm{off}} / L}{\tau_{p}-\tau_{r}},
$$

where $L$ is the rupture length. Note that we assume unit thickness of the fault. Figure 15 shows $D_{c}^{\text {eff }}$ as a function of rupture length with depth calculated from Figure $14 \mathrm{~b} . D_{c}^{\text {eff }}$ increases with the rupture length and with depth, implying more energy dissipation in the off-fault medium with large ruptures at depth. Up to half the amount of fracture energy on the main fault can be dissipated in the off-fault medium due to the coseismic off-fault damage at depth. 
a

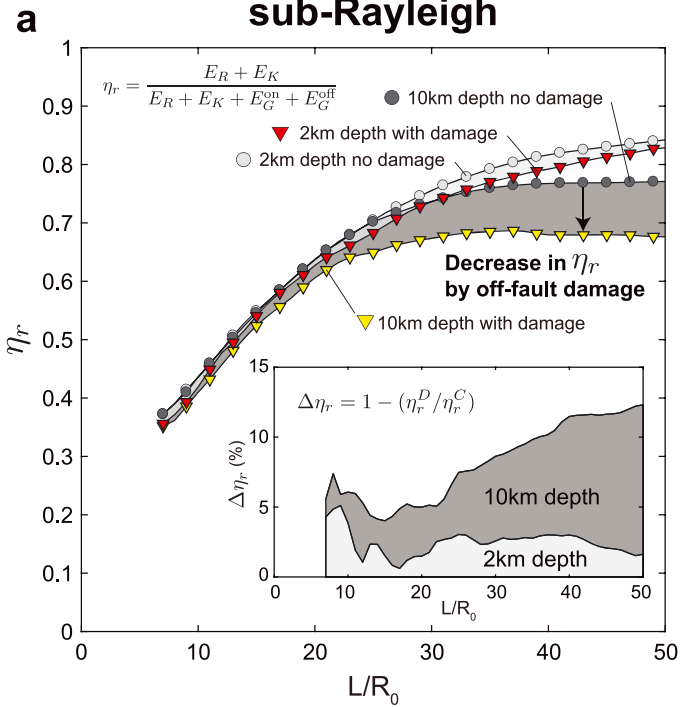

b supershear

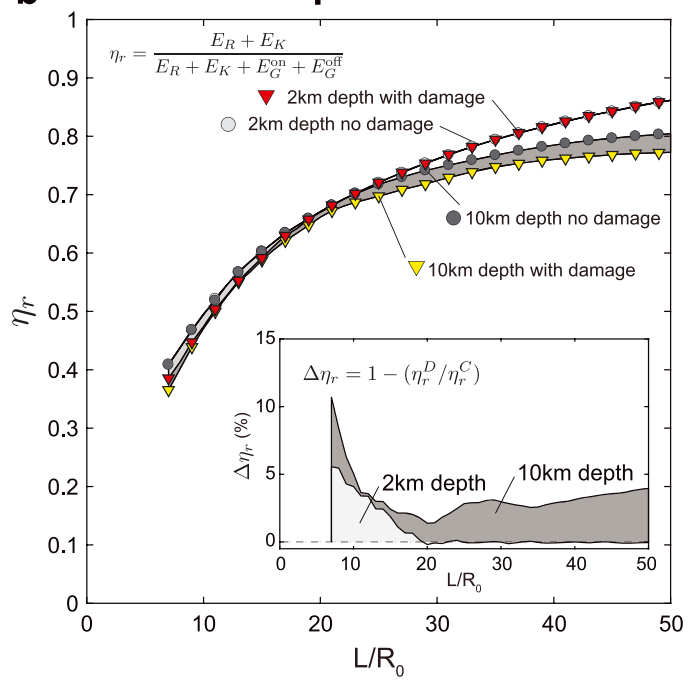

Figure 16. Seismic efficiency at 2- and 10-km depths with (a) $S=1.0$ and (b) $S=0.7$ as a function of rupture length. The circles indicate the cases without off-fault damage, whereas the inverted triangles indicate the cases with off-fault damage. The inset shows the percentage of the decrease in seismic efficiency due to the coseismic off-fault damage. Note that the rupture transitions to supershear around $L / R_{0}=40$ for the case without off-fault damage, while it remains sub-Rayleigh for the cases with off-fault damage in (a). The rupture transitions to supershear for both cases with and without off-fault damage in (b).

\subsection{Seismic Efficiency}

Seismic efficiency $\eta_{r}$ is an important parameter to quantify the proportion of radiated energy to the sum of radiated energy and fracture energy, which essentially evaluates the balance between the radiated energy as seismic waves and the dissipated energy due to on- and off-fault fracturing (Kanamori \& Brodsky, 2004). Since we can only evaluate the temporal radiated energy in equation (5) because of the infinite fault length in our model description, we define modified seismic efficiency for this study, given by

$$
\eta_{r}=\frac{E_{R}+E_{K}}{E_{R}+E_{K}+E_{G}^{\text {on }}+E_{G}^{\text {offf }}} .
$$

The physical interpretation of this quantity is same with the canonical seismic efficiency. We evaluated the evolution of $\eta_{r}$ as a function of rupture length at 2 - and $10-\mathrm{km}$ depths and compared between the cases with and without off-fault damage to investigate the effect of off-fault damage on the seismic efficiency. Figure 16 shows the $\eta_{r}$ for the cases with $S=1.0$ and $S=0.7$. The relative difference between the cases with and without off-fault damage is plotted in insets, defined as

$$
\Delta \eta_{r}=1-\frac{\eta_{r}^{D}}{\eta_{r}^{C}}
$$

where $\eta_{r}^{D}$ and $\eta_{r}^{C}$ indicate the $\eta_{r}$ with and without off-fault damage, respectively. There is a significant decrease in $\eta_{r}$ due to the coseismic off-fault damage, particularly in the deeper case with sub-Rayleigh rupture. This can be explained by the denser and more intricate off-fault fracture network formed at depth. Therefore, although the secondary off-fault fractures affect the high-frequency radiation, coseismic off-fault damage absorbs some of available energy, which would have been converted to radiated energy for the cases without off-fault damage.

\section{Conclusion}

Our systematic case study with depth demonstrated the mechanisms of coseismic off-fault fracturing and its effect on the rupture dynamics, the radiation, and the overall energy budget. The damage zone width decreases with depth, whereas the fracture density and the contribution of energy dissipation in off-fault medium globally increases with depth in nondimensional space. Overall, Figures 17a and 17b show the 

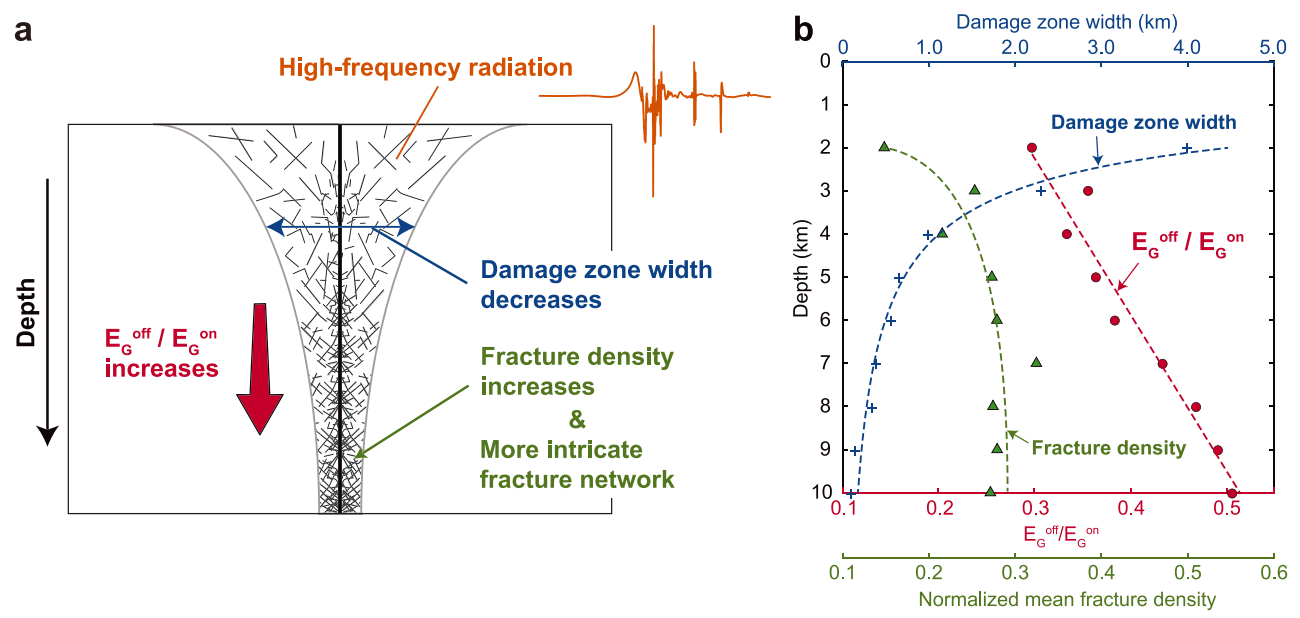

Figure 17. Evolution of damage zone width, fracture density, and contribution of off-fault damage to the overall energy budget. (a) Schematic of the off-fault damage with depth. (b) Damage zone width, fracture density, and the ratio of dissipated fracture energy in the off-fault medium to the energy dissipated on the main fault with depth. The markers indicate the values at examined depths. Solid lines indicate expected trends of the discrete data.

schematic of fault structure with depth based on this study and the summary of numerical results, inferred from the sub-Rayleigh cases $(S=1.0)$. The fracture density is evaluated using a representative value averaged over space with Figure 6. The damage zone width becomes narrower with depth, whereas the contribution of fracture energy to the overall energy budget rather increases with depth due to the increase in fracture density and complexity of off-fault fracture network.

In this study, we conducted simulations with intact rock and with fixed orientation of principal stress at $60^{\circ}$. Therefore, we observed the coseismic off-fault damage only in the extensional side of the fault. However, in nature, the off-fault damage is often observed on both sides of the fault. Due to the model constraints, and assumptions listed earlier, the present results, such as the damage zone width, do not always provide a close quantitative agreement to the observations.

The preexisting damage of the off-fault medium, the initial cohesion on the main fault, which is assumed to be zero in this study, and the orientation of the maximum principal stress also play a role in the off-fault damage on the compressional side. These need to be investigated by extensive parametric studies, which are outside the scope of this paper.

The present numerical framework is also applicable to natural fault system. Klinger et al. (2018) conducted dynamic earthquake rupture modeling of the 2016 Kaikoura earthquake with the same numerical framework as proposed in this study. They used the dynamic earthquake rupture modeling to resolve the most likely rupture scenario by comparing cosesmic off-fault damage pattern to the observations.

This study has opened an avenue to model dynamic earthquake ruptures with FDEM, which allows for modeling dynamic earthquake ruptures with explicit description of coseismic off-fault damage to better understand the fracturing mechanisms, the radiation, and the overall energy budget associated with earthquakes.

\section{Appendix A: Methodology for Modeling Coseismic Off-Fault Damage With FDEM}

Geological faults can be defined as discontinuities in a continuum medium. From this perspective, we consider both the faults and the off-fault damage as an aggregation of fractures at different length scales. FDEM is capable of modeling both continuum deformation and fracturing (i.e., dynamic rupture on the main fault and the off-fault damage) within the same numerical framework. In this appendix, we describe the essence of numerical framework for dynamic earthquake rupture modeling with coseismic off-fault damage. A set of detailed model formulation can be found in Okubo (2018). More details of main algorithmic solutions used within HOSSedu can be found in a series of monographs (Munjiza, 2004; Munjiza et al., 2011, 2015). 


\begin{tabular}{|c|c|c|}
\hline Variables & Description & Values \\
\hline$E^{\mathrm{a}}$ & Young's modulus & $75 \mathrm{GPa}$ \\
\hline$\mu^{\mathrm{a}}$ & Shear modulus & $30 \mathrm{GPa}$ \\
\hline$v^{\mathrm{a}}$ & Poisson's ratio & 0.25 \\
\hline$\rho^{\mathrm{a}}$ & Density & $2,700 \mathrm{~kg} / \mathrm{m}^{3}$ \\
\hline$\rho_{w}$ & Density of water & $1,000 \mathrm{~kg} / \mathrm{m}^{3}$ \\
\hline$\psi$ & Orientation of $\sigma_{1}$ & 60 \\
\hline$S$ & Seismic ratio & $0.7,1.0$ \\
\hline$d_{M C}$ & Closeness to failure & 0.4 \\
\hline$f_{s}$ & Static friction coefficient & 0.6 \\
\hline$f_{d}$ & Dynamic friction coefficient & 0.2 \\
\hline$D_{c}$ & Critical slip distance & Estimated from equation (A13) \\
\hline$G_{I I C}^{f * \mathrm{~b}}$ & Fracture energy on the main fault & $3 \mathrm{MJ} / \mathrm{m}^{2}$ \\
\hline$G_{I I C}^{f *, \text { off-fault b }}$ & Fracture energy in the off-fault medium & $0.01 \mathrm{MJ} / \mathrm{m}^{2}$ \\
\hline$C_{I}^{p \mathrm{c}}$ & Peak cohesion for tensile fractures & $8 \mathrm{MPa}$ \\
\hline$C_{I I}^{p}$ & Peak cohesion for shear fractures & Estimated from equation (A15) \\
\hline
\end{tabular}

\section{A1. Initial Stress State at Depth}

We follow a similar process to that proposed by Templeton and Rice (2008) and Xu et al. (2012) in order to make an assumption of initial stress state as a function of depth. The initial stress state is set for triggering right-lateral strike-slip on the main fault. The initial stress state is uniform in the homogeneous and isotropic elastic medium, given by

$$
\sigma_{i j}^{0}=\left[\begin{array}{cc}
\sigma_{x x}^{0} & \sigma_{y x}^{0} \\
\sigma_{y x}^{0} & \sigma_{y y}^{0}
\end{array}\right] .
$$

Note that we assume plane strain conditions. Let normal stress $\sigma_{y y}^{0}$ on the main fault be given by linear overburden effective stress gradient as it provides an approximation of the magnitude of normal stress on the main fault with depth, such that

$$
\sigma_{y y}^{0}=-\left(\rho-\rho_{w}\right) g z
$$

where $\rho$ is the density of rock, $\rho_{w}$ is the density of water, $g$ is the gravitational acceleration and $z$ is the depth measured from the ground surface. The initial shear stress $\sigma_{y x}^{0}$ is estimated in terms of the seismic $S$ ratio, defined by Andrews (1976), on the main fault such as

$$
S=\frac{f_{s}\left(-\sigma_{y y}^{0}\right)-\sigma_{y x}^{0}}{\sigma_{y x}^{0}-f_{d}\left(-\sigma_{y y}^{0}\right)},
$$

where $f_{s}$ and $f_{d}$ are the static and dynamic friction coefficients, respectively. The $S$ ratio defines whether the rupture transitions to supershear $(S<1.77)$ or remains sub-Rayleigh $(S>1.77)$ with 2-D purely elastic model (i.e., no off-fault damage). From equation (A3), the initial shear stress on the main fault can be derived as

$$
\sigma_{y x}^{0}=\frac{f_{S}+S f_{d}}{1+S}\left(-\sigma_{y y}^{0}\right)
$$

The horizontal compressive stress $\sigma_{x x}^{0}$ is then determined by the normal stress $\sigma_{y y}^{0}$, shear stress $\sigma_{y x}^{0}$ and the given orientation of initial compressive principal stress to the main fault $\psi$ as follows:

$$
\sigma_{x x}^{0}=\left(1-\frac{2 \sigma_{y x}^{0}}{\tan (2 \psi) \sigma_{y y}^{0}}\right) \sigma_{y y}^{0} .
$$




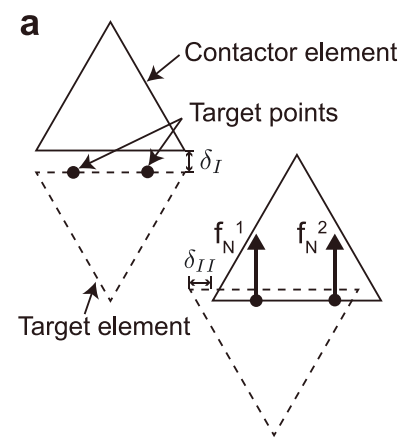

C

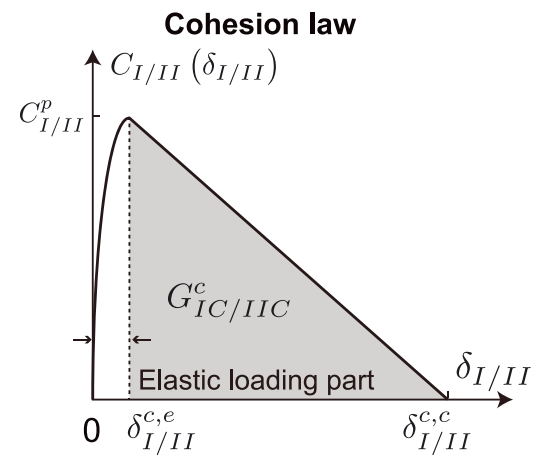

b
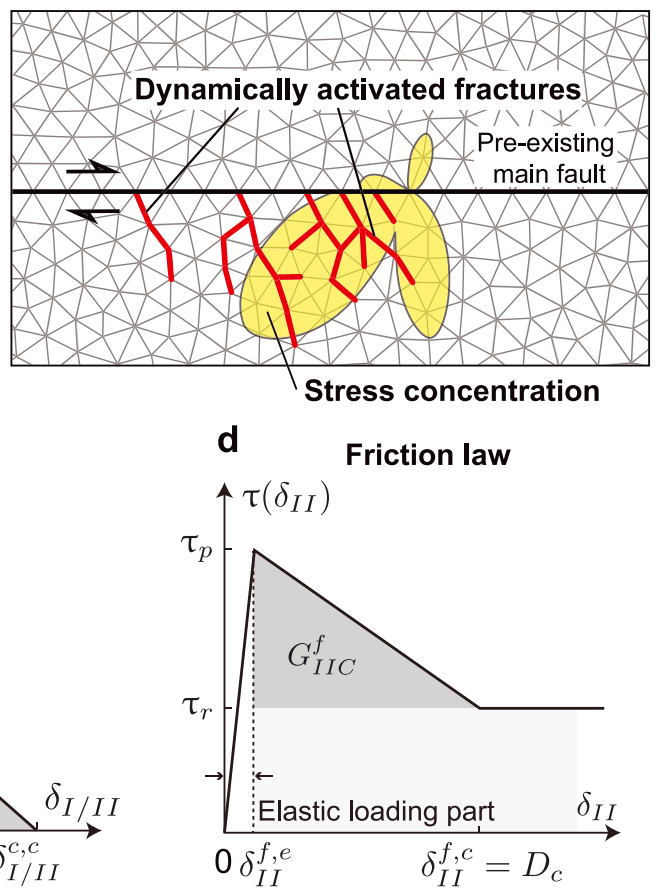

Figure A1. Numerical framework of FDEM for dynamic earthquake rupture modeling. (a) Schematic for contactor and target. Opening displacement $\delta_{I}$, shear displacement $\delta_{I I}$, and contact forces $f_{N}$ are indicated. The number of target points indicated by black dots is properly chosen for required numerical accuracy. (b) Model description showing the mesh discretization and the off-fault fractures. Computational domain is discretized using an unstructured mesh. Every interface between elements is regarded as a potential failure plane, where cohesion and friction are operating as a function of $\delta_{I / I I}$. When the tensile or shear cohesion starts weakening, we plot the interface as secondarily activated fractures as shown in red lines. (c) Linear displacement softening law for cohesion. The area highlighted in gray under the softening part of the curve indicates the fracture energy associated with cohesion in tension $G_{I C}^{c}$ and in shear $G_{I I C}^{c}$, respectively. (d) Linear slip-weakening law for friction. The energy dissipated by frictional process is divided into the fracture energy associated with friction $G_{I I C}^{f}$, while the rest is considered as heat energy.

\section{A2. Damage Type and Fracture Energy}

In the FDEM framework, fractures are represented as the loss of cohesion at the interfaces of finite elements. The cohesion and the friction between the contactor and the target elements are represented as a function of relative displacements defined by the aperture $\delta_{I}$ and the slip $\delta_{I I}$ (Figure A1a). Figure A1b shows the mesh discretization and the schematic for off-fault fractures. The cohesive and frictional resistances are applied on every interface between elements, regarded as a potential failure plane. Fractures are activated when the cohesion starts to be broken due to the stress concentration of the dynamic earthquake rupture. Both cohesion and friction curves are divided into two parts, an elastic loading part and a displacement-weakening part as shown in Figures A1c and A1d. In the elastic loading part, the resistant forces against the displacements acting on the interface increase quadratically (for the case of cohesion) or linearly (for the case of friction) with the stiffness of elastic loading portions. Since this elastic loading part ideally should be zero to represent the material continuity, the stiffnesses are chosen to be much higher than Young's modulus of the material to minimize the displacements associated with the elastic loading.

When the traction applied on the interface reaches the peak cohesion for tensile fractures $C_{I}^{p}$ or for shear fractures $C_{I I}^{p}$, the cohesion starts weakening, and eventually it is totally broken, behaving as a secondarily activated fracture (Figure A1c). The friction curve follows linear slip-weakening law, originally proposed by Ida (1972) and Palmer and Rice (1973), which has been widely used for dynamic earthquake rupture modeling (e.g., Andrews, 1976; Aochi \& Fukuyama, 2002; De La Puente et al., 2009). When the shear traction reaches to frictional strength $\tau_{p}$, it decreases down to the residual strength $\tau_{r}$ at critical slip distance for friction $\delta_{I I}^{f, c}=D_{c}$ as shown in Figure A1d. $\tau_{p}$ and $\tau_{r}$ are defined as

$$
\tau_{p}=f_{s}\left(-\sigma_{n}\right)
$$




$$
\tau_{r}=f_{d}\left(-\sigma_{n}\right),
$$

where $\sigma_{n}$ is the normal stress on the contact interface. Note that the friction law is operating both on the main fault and the secondary fractures activated in the off-fault medium. The residual traction on the fracture surface is zero for tensile fractures as long as the damage on the fracture surface is equal to one and as long as the fracture remains open, while the residual shear traction is kept at $\tau_{r}$ for shear fractures even after the shear cohesion is broken.

The mixed mode fracture is evaluated by a damage parameter, $D$, which is defined as

$$
\begin{gathered}
D_{i}=\frac{\delta_{i}-\delta_{i}^{c, e}}{\delta_{i}^{c, c}-\delta_{i}^{c, e}} \quad i=I, I I \\
D=\sqrt{D_{I}^{2}+D_{I I}^{2}} \quad(0 \leq D \leq 1) \\
D^{T}=\frac{D_{I}}{D}=\left\{\begin{array}{l}
1, \text { for purely tensile fracture } \\
0, \text { for purely shear fracture }
\end{array}\right\},
\end{gathered}
$$

where $D_{i}(i=I, I I)$ is the components of damage for tensile and shear fractures, $\delta_{i}$ is normal and tangential displacement, $\delta_{i}^{c, e}$ is the initial critical displacement for elastic loading, $\delta_{i}^{c, c}-\delta_{i}^{c, e}$ is the maximum displacement during linear softening, where $\delta_{i}^{c, c}$ is the initial critical displacement for linear-weakening part, $D$ is the degree of damage, and $D^{T}$ indicates the type of damage. Similar expressions can be found in Rougier et al. (2011) and Lisjak et al. (2014).

Since we used a linear softening law, the fracture energy associated with the cohesion for tensile (Mode I) and shear (Mode II) fractures $G_{I C / I I C}^{c}$ (i.e., the energy required to completely break the connection of the contact) is evaluated as

$$
G_{i C}^{c}=\frac{1}{2} C_{i}^{p}\left(\delta_{i}^{c, c}-\delta_{i}^{c, e}\right) \quad i=I, I I .
$$

The fracture energy for friction $G_{I I C}^{f}$ is, following Palmer and Rice (1973), described as

$$
G_{I I C}^{f}=\frac{1}{2} D_{c}\left(\tau_{p}-\tau_{r}\right)
$$

Note that the elastic loading part for friction $\delta_{I I}^{f, e}$ is much smaller than $D_{c}$, so that the representation of fracture energy $G_{I I C}^{f}$ by equation (A12) is acceptable even without the consideration of elastic loading part.

In this study, we assume that the fracture energy on the main fault is kept constant with depth, denoted as $G_{I I C}^{f *}$. Thus, $D_{c}$ decreases with depth as follows:

$$
D_{c}(z)=\frac{2 G_{I I C}^{f *}}{\left(f_{s}-f_{d}\right)\left\{-\sigma_{y y}^{0}(z)\right\}} .
$$

$\delta_{i}^{c, e}$ and $\delta_{i}^{c, c}(i=I, I I)$ are derived with the stiffness of elastic loading part and a given fracture energy. The shear fracture energy is estimated from experiments and observations, following the scaling law between the fracture energy and the amount of slip (Passelègue et al., 2016; Viesca \& Garagash, 2015). It provides a reasonable assumption of fracture energy on the fault and in the off-fault medium, corresponding to the mean slip on the main fault and on the off-fault fractures during the ruputure process. The fracture energy on the main fault is assigned to be 2 orders of magnitude higher than that of individual off-fault fractures because the slip on the main fault is larger than that of the off-fault fractures when modeling with a single planar fault. We assume $\delta_{i}^{c, e}=\delta_{i}^{f, e}$ so that the cohesion and the friction start weakening at the same amount of slip. The detailed formulations can be found in Okubo (2018, Chapter 2). 


\section{A3. Parametrization for Peak Cohesions}

To determine $C_{I I}^{p}$, we used the closeness to failure $d_{\mathrm{MC}}$, which indicates the safety of the initial stress state to the failure of the material represented by the ratio of the radius of Mohr's circle to the distance to the Mohr-Coulomb criteria (see also Templeton \& Rice, 2008). Let $\sigma_{1}$ and $\sigma_{2}$ be the maximum and minimum compressive principal stresses. Then $d_{\mathrm{MC}}$ is derived from geometrical relationships as

$$
\begin{aligned}
d_{\mathrm{MC}} & =\frac{\sigma_{2}-\sigma_{1}}{2 C_{I I}^{p} \cos \phi-\left(\sigma_{1}+\sigma_{2}\right)} \\
& =\frac{\left(\frac{\sigma_{1}}{\sigma_{2}}-1\right)}{\left(\frac{\sigma_{1}}{\sigma_{2}}+1\right)-2\left(\frac{C_{I I}^{p}}{\sigma_{2}} \cos \phi\right)},
\end{aligned}
$$

where $\phi$ is the friction angle as $\tan \phi=f_{s} . d_{\mathrm{MC}}<1$ means no failure and $d_{\mathrm{MC}} \geq 1$ implies the initiation of failure in shear on the corresponding plane. Note that $d_{\mathrm{MC}}$ locally changes due to perturbations of the stress field.

In the case study, initial $d_{\mathrm{MC}}$ is kept constant with depth for the fair comparison between the different stress states. By assuming the constant orientation of maximum compressive principal stress $\Psi$ and seismic ratio $S$, the ratio of principal stresses $\sigma_{1} / \sigma_{2}$ is also kept constant with depth. Thus, from equation (A14), the ratio $C_{I I}^{p} / \sigma_{2}$ has to be kept constant to obtain an equal closeness to failure with depth, implying $C_{I I}^{p}$ must increase linearly with depth. Therefore, we first calculate $\sigma_{i j}^{0}$ as described in section A1, and then we derive $C_{I I}^{p}$ as

$$
C_{I I}^{p}=\frac{\sigma_{2}-\sigma_{1}+d_{\mathrm{MC}}\left(\sigma_{1}+\sigma_{2}\right) \sin \phi}{2 d_{\mathrm{MC}} \cos \phi}
$$

where $d_{\mathrm{MC}}$ should be chosen carefully to avoid $C_{I I}^{p}$ being negative. $C_{I}^{p}$ is chosen from the experiments (Cho et al., 2003), kept constant with depth. We assume the acceptable range for $C_{I}^{p}$ is between 1 and $10 \mathrm{MPa}$. Since peak tensile and shear values of cohesion play an important role in the activation of off-fault fracture networks, we need to investigate the effect of initial peak cohesion values on the high-frequency radiation and the overall energy budget.

\section{A4. Process Zone Size}

The quasi-static process zone size $R_{0}$ is used to nondimensionalize length scale as it characterizes the scale of dynamic earthquake ruptures (Poliakov et al., 2002; Rice et al., 2005), described as

$$
R_{0}(z)=\frac{9 \pi}{16(1-v)} \frac{\mu G_{I I C}^{f}}{\left[\left(f_{s}-f_{d}\right)\left\{-\sigma_{y y}^{0}(z)\right\}\right]^{2}},
$$

where $v$ is Poisson's ratio and $\mu$ is shear modulus. $R_{0}(z)$ decreases with depth as a function of $\left\{-\sigma_{y y}^{0}(z)\right\}^{-2}$. Since the size of potential failure area is of the same order of magnitude as $R_{0}(z)$ in the analysis with steady-state crack (Poliakov et al., 2002), the damage zone size is also expected to decrease when assuming constant $G_{I I C}^{f}$ with depth. Although we model a spontaneous rupture propagation, the results of flower-like structure as shown in Figure 8 can be explained by this estimation as the rupture velocity for the case of sub-Rayleigh rupture $(S=1.0)$ with off-fault damage converges to slightly below of its limiting speed (Figure 10). The case study for constant Dc with depth also shows the damage zone width decreasing with depth, which can be found in Okubo (2018, Chapter 3).

The dynamic process zone size $R_{f}\left(v_{r}\right)$ is generally inversely proportional to the rupture velocity $v_{r}$, given by Rice, (1980, Equation 6.16) and Freund (1990, Equation 6.2.35). $R_{f}\left(v_{r}\right)$ gradually shrinks and asymptotically converges to 0 as the rupture velocity approaches its limiting speed, which is known as Lorentz contraction.

\section{A5. Potential Failure Area}

We superimposed the stress concentration in Figure 5 to highlight the potential failure area, where the secondary fractures are likely to be activated. For tensile fracture, we used the normalized first stress invariant

$$
\frac{I_{1}(t)}{I_{1}^{\text {init }}}=\frac{\sigma_{k k}(t)}{\sigma_{k k}^{0}},
$$


where $I_{1}^{\text {init }}=I_{1}(0)$ and $\sigma_{k k}=\sigma_{x x}+\sigma_{y y}+\sigma_{z z}$, where $\sigma_{z z}=v\left(\sigma_{x x}+\sigma_{y y}\right)$ under plane strain conditions. The small $I_{1}(t) / I_{1}^{\text {init }}$ thus indicates less confining pressures. For shear fracture, we used the normalized closeness to failure $d_{\mathrm{MC}} / d_{\mathrm{MC}}^{\text {init }}$. The large $d_{\mathrm{MC}} / d_{\mathrm{MC}}^{\text {init }}$ indicates that the stress state is close to shear failure.

\section{Appendix B: Cross Validation of 2-D FDEM for Earthquake Rupture Modeling}

We performed cross validation of the FDEM-based software tool, HOSSedu (denoted as HOSS in this section), to assess the achievable accuracy of dynamic earthquake rupture modeling with purely elastic medium, that is, no off-fault damage, by comparing the results with HOSS to those with other numerical schemes. We chose the finite difference method (FDM), the spectral element method (SEM), and the boundary integral equation method (BIEM) as comparison basis as they have been verified in previous studies (e.g., Day et al., 2005; Kaneko et al., 2008; Koller et al., 1992).

The cross-validation effort for HOSS is based on a similar process to Kaneko et al. (2008). The first arrival time of the rupture is used to evaluate the numerical precision of the rupture solution (Day et al., 2005). In this study, the rupture arrival time is defined when the shear traction reaches the peak strength $\tau_{p}$. We followed the Version 3 of the benchmark problem proposed by the Southern California Earthquake Center/U.S. Geological Survey dynamic earthquake rupture code verification exercise (Harris et al., 2009), commonly used for cross-validating numerical schemes (Day et al., 2005; De La Puente et al., 2009; Kaneko et al., 2008; Rojas et al., 2008). The model is originally described in 3-D so that the 2-D analog model was used in this study, similar to Rojas et al. (2008), Kaneko et al. (2008), and De La Puente et al. (2009).

Figure B1a shows the comparison of slip velocity history at $x=9 \mathrm{~km}$ from the center of the main fault. The results of HOSS are compared to FDM, SEM, and BIEM, where the grid spacing on the fault $\Delta s$ is chosen for the highest resolution as $\Delta s=8 \mathrm{~m}\left(R_{0} / \Delta s=116\right)$ for HOSS, FDM, and BIEM and $\Delta s=10 \mathrm{~m}\left(R_{0} / \Delta s=93\right)$ for SEM. The slip velocity history of HOSS is consistent with the other numerical schemes except for the peak slip velocity. The peak slip velocity of HOSS is $4.1 \%$ smaller than that of BIEM, and the rupture arrival time is slightly faster than the others. Both of the small differences are explained by the artificial viscous damping. There is no viscous damping for BIEM and FDM, whereas the Kelvin-Voigt viscous damping is used for SEM, and the Munjiza viscosity is used for HOSS. Although the viscous damping causes a small decrease in the peak slip velocity and shortens the rupture arrival time, the high-frequency numerical noise is significantly removed for the result with HOSS. It is notable that the comparison of HOSS to BIEM is no longer fair due to the artificial viscous damping, so that the evaluation of the effect of viscous damping on the rupture propagation is worthwhile, discussed later in this section.

Figure B1b shows the grid convergence of HOSS and the others. The numerical accuracy as a function of grid resolution is evaluated by the root-mean-square (RMS) difference. The RMS error of the rupture arrival time is calculated by the comparison to the benchmark solution provided by BIEM with highest resolution as it is semianalytical solution. Although the RMS error is slightly higher than the FDM and SEM due to the viscosity, the convergence rate of HOSS is similar to BIEM, following the power law with the scaling exponent of 1.6 for HOSS and 1.4 for BIEM. Thus, the numerical accuracy is assured with appropriate $\Delta s$ for the required error range of earthquake rupture modeling.

Figures B1c and B1d show the RMS error of the rupture arrival time with various viscous values, grid resolutions and the number of points per edge. The circles indicate the examined combinations of viscosity and grid resolution, where the size of circles with monochromatic gradation represents the proportion of the viscosity to the theoretically derived critical viscosity (see also Okubo, 2018). The saddle of the RMS error around $\eta / M_{v} \Delta t=10^{2}$, where $\eta$ is viscosity, $M_{v}$ is the Munjiza constant, and $\Delta t$ is time step, is explained by the trade-off between the numerical oscillation and the overdamping. The convergence of RMS error is better with two integral points per edge. Hence, the grid resolution, viscosity, and the number of points per face should be carefully chosen for the required numerical accuracy. Since the number of points per edge has to be more than 2 in order to allow for the secondary fractures in the off-fault medium due to numerical reasons, we chose the appropriate grid size and viscosity from Figure B1d for the case study with depth.

\section{Appendix C: Mesh Dependency}

We examined two types of mesh to investigate mesh dependency associated with the coseismic off-fault damage. We made a mesh \#1, which is used for the case study in the main section, and another mesh \#2, 

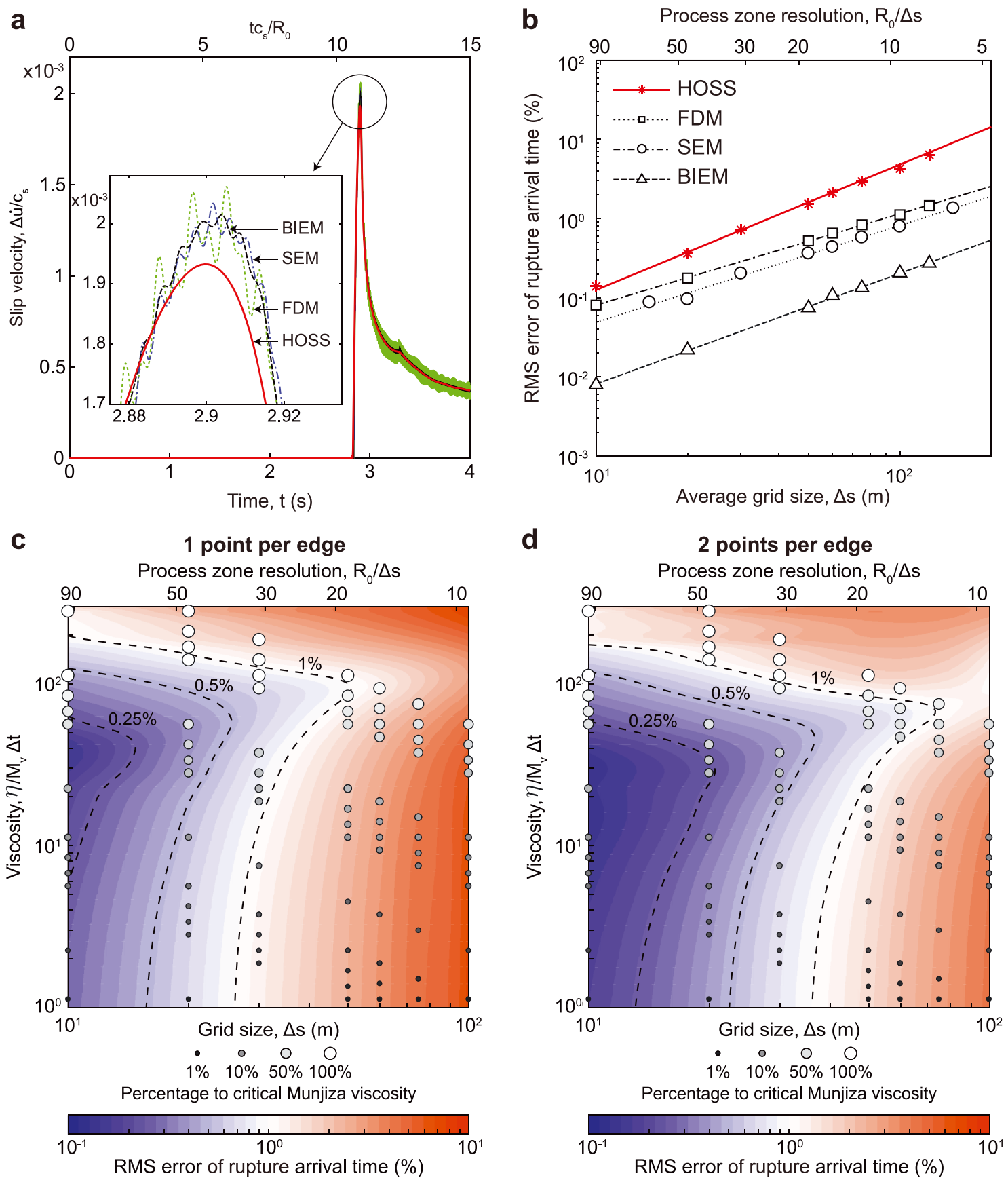

Figure B1. Summary of the cross validation of HOSS. (a) Slip velocity history at $x=9.0 \mathrm{~km}\left(x / R_{0}=9.7\right)$. (b) Grid convergence as a function of grid size. The RMS error is calculated by the comparison of rupture arrival time to the benchmark result provided by the solution of BIEM with highest resolution. The HOSS simulations are performed with two points per edge. (c, d) RMS error of the rupture arrival time with various combinations of viscosity and grid size with (c) one point and (d) two points per edge. The circles indicate the examined combinations, where the size of circles with monochromatic gradation represents the proportion of viscosity to the critical viscosity (the viscosity is higher with light color and with large circle). Color contour indicates the RMS error of rupture arrival time obtained by interpolating the examined combinations. BIEM = boundary integral equation method; HOSS $=$ Hybrid Optimization Software Suite-Educational; RMS = root-mean-square. 
a

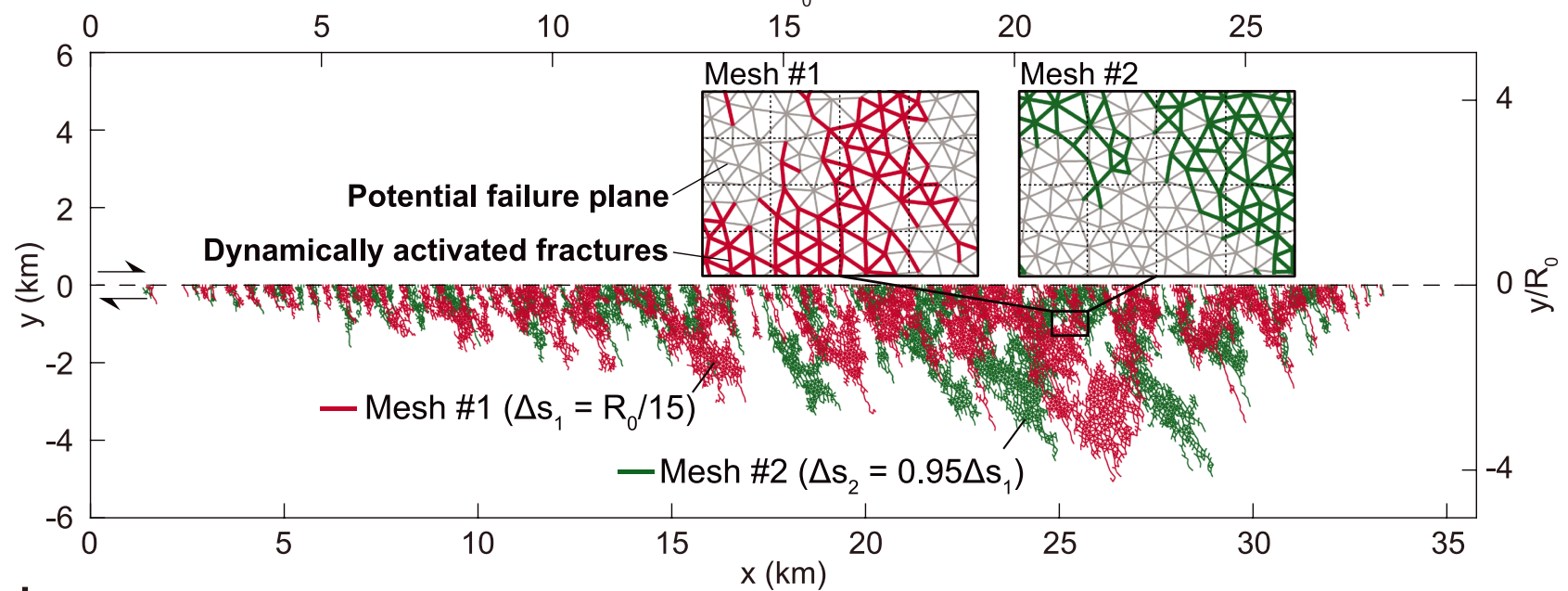

b

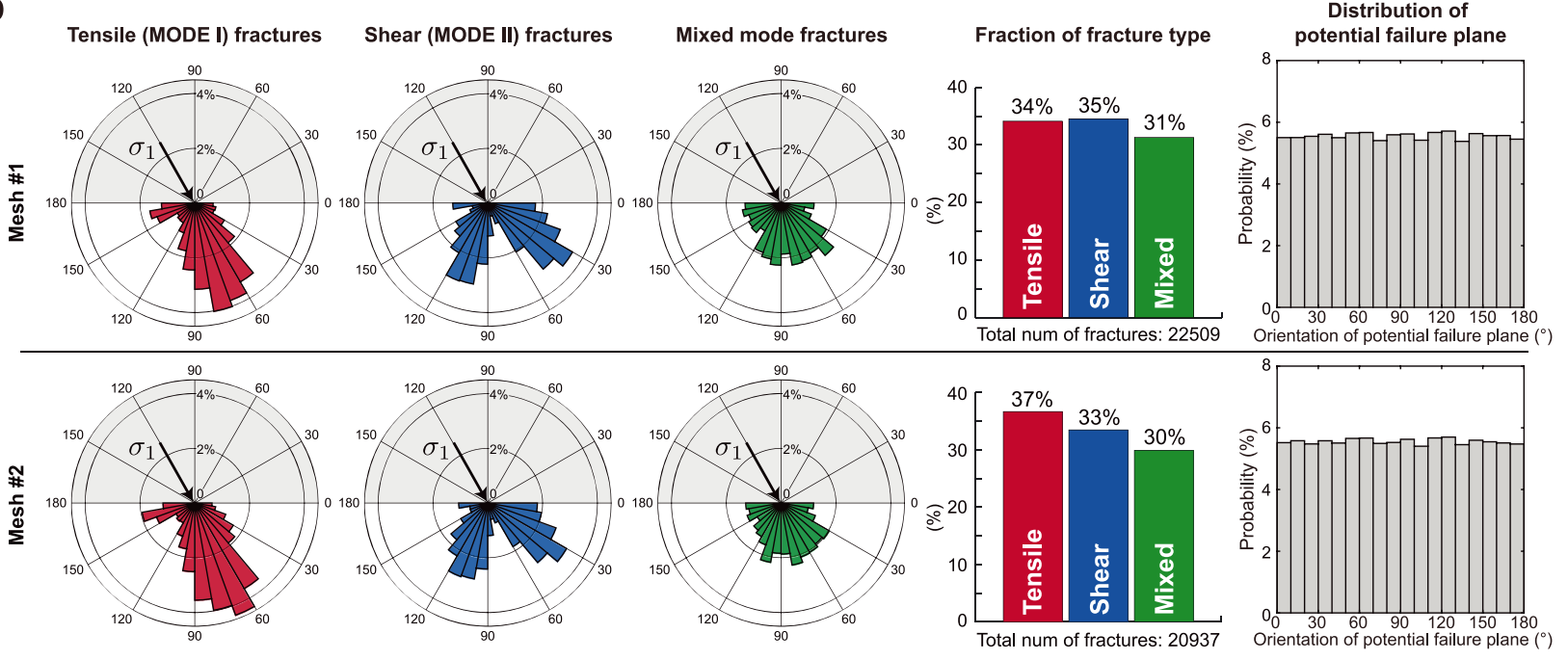

Figure C1. Comparison of off-fault damage pattern. (a) Trace of off-fault fractures with different meshes. (b) Rose diagram of the orientation of off-fault fractures, fraction of damage type, and distribution of potential failure planes.

where the grid size on the fault is $5 \%$ smaller than the mesh $\# 1$ in order to change the mesh topology. We conducted a dynamic rupture simulation at 2-km depth with $S=1.0$ using these meshes. Figure C1a shows the trace of off-fault fractures with each mesh. The damage zone width is consistent between them, whereas the damage pattern varies due to the different arrangement of potential failure planes in the off-fault medium. Small perturbation in the mesh topology thus changes the detailed damage pattern because of its chaotic aspects of the system.

However, statistical quantities are not influenced by the mesh topology. Figure C1b shows the rose diagram of the orientation of off-fault fractures, which is in agreement between mesh \#1 and mesh \#2. In addition, the fraction of fracture type is also compatible between them using the meshes, where the orientation of potential failure plane is uniformly averaged as shown in the histogram. The authors realize that there is a need to further explore the evolution and convergence of statistical quantities with mesh size in off-fault medium; however, this work is outside the scope of this paper.

Figure C2a shows the spatial distribution of critical frequency and spectra associated with the meshes. The spatial distribution varies as it depends on the damage pattern, whereas both spectra show the enhanced high-frequency radiation regardless of mesh topology. Figure $\mathrm{C} 2 \mathrm{~b}$ shows the comparison in the seismic efficiency $\eta_{r}$ and the contribution of fracture energy associated with the off-fault damage $E_{G}^{\text {off }} / E_{G}^{\text {on }}$ as a function of time. $\eta_{r}$ is well consistent between the meshes. Since we indirectly evaluate the $E_{G}^{\text {off }}$ with the uncertainty 

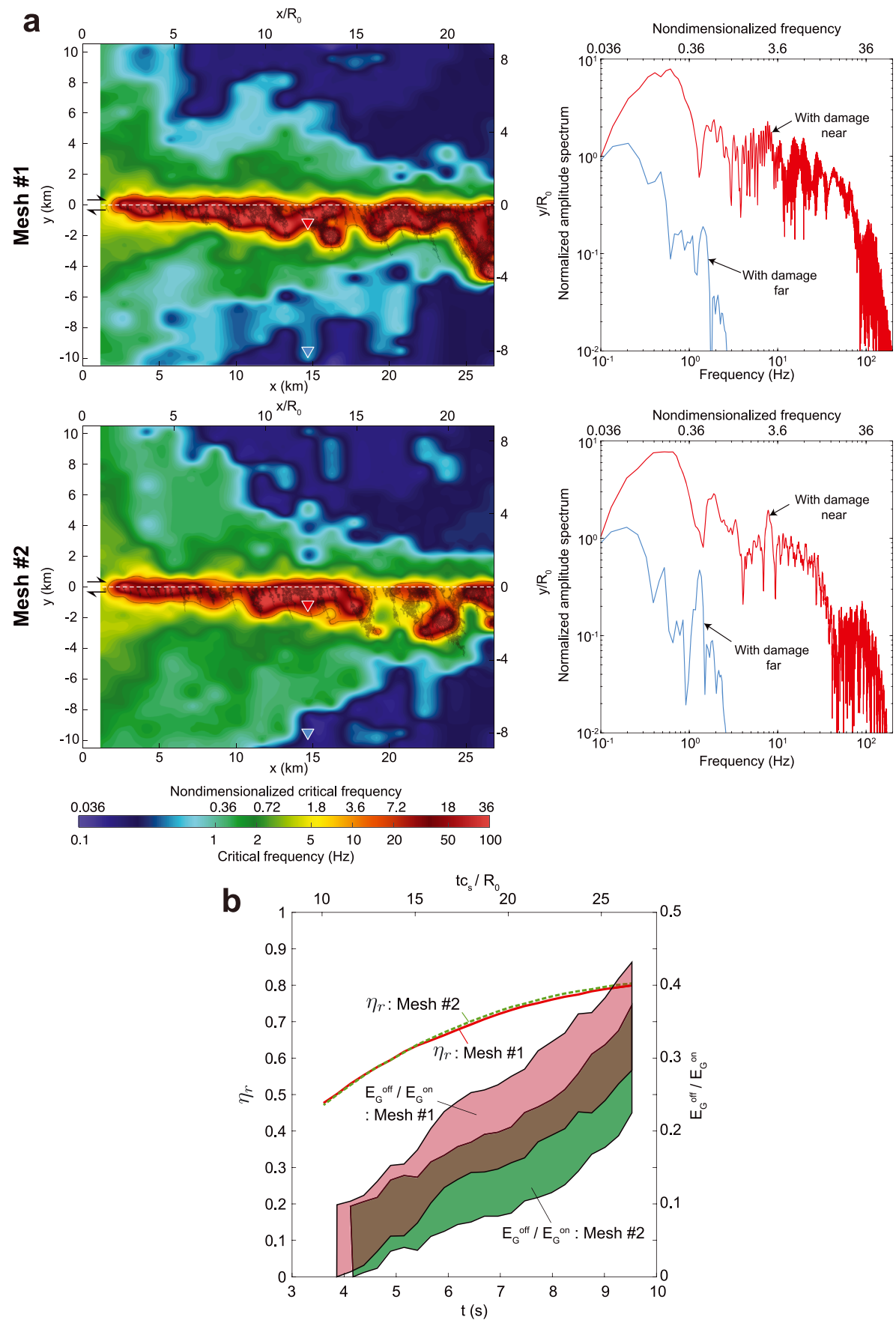

Figure C2. Radiation and overall energy budget with different meshes. (a) Distribution of critical frequency in space and spectra at near and far from the main fault. (b) Seismic efficiency $\eta_{r}$ and the fraction of $E_{G}^{\text {off }}$ to $E_{G}^{\text {on }}$. The bands indicate the estimation of $E_{G}^{\text {off }} / E_{G}^{\text {on }}$ with the uncertainty of $\pm 15 \%$ in energy dissipation due to the numerical viscous damping. 
of energy dissipation due to the numerical viscous damping, the estimation of $E_{G}^{\text {off }} / E_{G}^{\text {on }}$ is shown with error bands. Both results are fairly overlapped and show the increase in $E_{G}^{\mathrm{off}} / E_{G}^{\mathrm{on}}$ with rupture propagation, which is sufficient for the argument in section 6.

In summary, although the detailed damage pattern depends on the mesh topology, the statistical quantities such as orientation of fractures, radiation and overall energy budget are not so much influenced. Furthermore, when considering geometrical complexity of the fault system, the damage pattern is dominantly determined by the stress concentration caused by the fault geometry, such as fault kinks or fault roughness, rather than the mesh topology.

\section{Acknowledgments}

The authors would like to thank Yehuda Ben-Zion and two anonymous reviewers for their constructive reviews. We also thank Raúl Madariaga for his insightful feedbacks. The cross validation of HOSSedu was performed using the softwares for dynamic earthquake rupture modeling; MultiDimensional Spectral Boundary Integral code (MDSBI), available at https://pangea.stanford. edu/ edunham/codes/codes.html and spectral element method tool for 2-D wave propagation and earthquake source dynamics (SEM2DPACK), available at http://web.gps.caltech. edu/ ampuero/software.html. The mesh discretization was performed with the mesh generation toolkit, Trelis Pro. We acknowledge Los Alamos National Laboratory (LANL) Institutional Computing program for the computing resources provided for this work. Part of numerical simulation was also performed on the S-CAPAD platform, IPGP. This work is supported by the LANL LDRD Program (20170004DR), the PhD funding from Université Sorbonne Paris Cité (USPC) and the European Research Council grant REALISM (2016-grant 681346).

\section{References}

Aki, K., \& Richards, P. G. (2002). Quantitative seismology. Sausalito, CA: University Science Books.

Ando, R., \& Yamashita, T. (2007). Effects of mesoscopic-scale fault structure on dynamic earthquake ruptures: Dynamic formation of geometrical complexity of earthquake faults. Journal of Geophysical Research, 112, B09303. https://doi.org/10.1029/2006JB004612

Andrews, D. J. (1976). Rupture velocity of plane strain shear cracks. Journal of Geophysical Research, 81(B32), 5679-5689. https://doi.org/ 10.1029/JB081i032p05679

Andrews, D. J. (1985). Dynamic plane-strain shear rupture with a slip-weakening friction law calculated by a boundary integral method. Bulletin of the Seismological Society of America, 75, 1-21.

Andrews, D. J. (2005). Rupture dynamics with energy loss outside the slip zone. Journal of Geophysical Research, 110, B01307. https://doi. org/10.1029/2004JB003191

Aochi, H., \& Fukuyama, E. (2002). Three-dimensional nonplanar simulation of the 1992 Landers earthquake. Journal of Geophysical Research, 107(B2), 2035. https://doi.org/10.1029/2000JB000061

Ben-Zion, Y., \& Shi, Z. (2005). Dynamic rupture on a material interface with spontaneous generation of plastic strain in the bulk. Earth and Planetary Science Letters, 236(1-2), 486-496. https://doi.org/10.1016/j.epsl.2005.03.025

Bhat, H. S., Rosakis, A. J., \& Sammis, C. G. (2012). A micromechanics based constitutive model for brittle failure at high strain rates. Journal of Applied Mechanics, 79(3), 031016. https://doi.org/10.1115/1.4005897

Cappa, F., Perrin, C., Manighetti, I., \& Delor, E. (2014). Off-fault long-term damage: A condition to account for generic, triangular earthquake slip profiles. Geochemistry, Geophysics, Geosystems, 15, 1476-1493. https://doi.org/10.1002/2013GC005182

Castro, R. R., \& Ben-Zion, Y. (2013). Potential signatures of damage-related radiation from aftershocks of the 4 April 2010 (Mw 7.2) El Mayor-Cucapah Earthquake, Baja California, México. Bulletin of the Seismological Society of America, 103(2A), 1130-1140. https://doi. org/10.1785/0120120163

Chester, F. M., Evans, J. P., \& Biegel, R. L. (1993). Internal structure and weakening mechanisms of the San Andreas fault. Journal of Geophysical Research, 98, 771-786. https://doi.org/10.1029/92JB01866

Chester, F. M., \& Logan, J. M. (1986). Implications for mechanical properties of brittle faults from observations of the Punchbowl fault zone, California. Pure and Applied Geophysics, 124(1-2), 79-106.

Cho, S. H., Ogata, Y., \& Kaneko, K. (2003). Strain-rate dependency of the dynamic tensile strength of rock. International Journal of Rock Mechanics and Mining Sciences, 40(5), 763-777. https://doi.org/10.1016/S1365-1609(03)00072-8

Cochran, E. S., Li, Y.-G., Shearer, P. M., Barbot, S., Fialko, Y., \& Vidale, J. E. (2009). Seismic and geodetic evidence for extensive, long-lived fault damage zones. Geology, 37(4), 315-318. https://doi.org/10.1130/G25306A

Dalguer, L. A., Irikura, K., \& Riera, J. D. (2003). Simulation of tensile crack generation by three-dimensional dynamic shear rupture propagation during an earthquake. Journal of Geophysical Research, 108(B3), 2144. https://doi.org/10.1029/2001JB001738

Day, S. M., Dalguer, L. A., Lapusta, N., \& Liu, Y. (2005). Comparison of finite difference and boundary integral solutions to three-dimensional spontaneous rupture. Journal of Geophysical Research, 110, B12307. https://doi.org/10.1029/2005JB003813

De La Puente, J., Ampuero, J.-P., \& Käser, M. (2009). Dynamic rupture modeling on unstructured meshes using a discontinuous Galerkin method. Journal of Geophysical Research, 114, B10302. https://doi.org/10.1029/2008JB006271

Dunham, E. M., Belanger, D., Cong, L., \& Kozdon, J. E. (2011a). Earthquake ruptures with strongly rate-weakening friction and off-fault plasticity, Part 1: Planar faults. Bulletin of the Seismological Society of America, 101(5), 2296-2307. https://doi.org/10.1785/0120100075

Dunham, E. M., Belanger, D., Cong, L., \& Kozdon, J. E. (2011b). Earthquake ruptures with strongly rate-weakening friction and off-fault plasticity, Part 2: Nonplanar faults. Bulletin of the Seismological Society of America, 101(5), 2308-2322. https://doi.org/10.1785/ 0120100076

Faulkner, D. R., Mitchell, T. M., Jensen, E., \& Cembrano, J. (2011). Scaling of fault damage zones with displacement and the implications for fault growth processes. Journal of Geophysical Research, 116, B05403. https://doi.org/10.1029/2010JB007788

Fletcher, J. M., Teran, O. J., Rockwell, T. K., Oskin, M. E., Hudnut, K. W., Mueller, K. J., et al. (2014). Assembly of a large earthquake from a complex fault system: Surface rupture kinematics of the 4 April 2010 El Mayor-Cucapah (Mexico) Mw 7.2 earthquake. Geosphere, 10(4), 797-827. https://doi.org/10.1130/GES00933.1

Freund, L. B. (1990). Dynamic fracture mechanics. Cambridge: Cambridge University Press.

Fukuyama, E. (2005). Radiation energy measured at earthquake source. Geophysical Research Letters, 32, L13308. https://doi.org/10.1029/ 2005GL022698

Gabriel, A.-A., Ampuero, J.-P., Dalguer, L., \& Mai, P. M. (2013). Source properties of dynamic rupture pulses with off-fault plasticity. Journal of Geophysical Research: Solid Earth, 118, 4117-4126. https://doi.org/10.1002/jgrb.50213

Hanks, T. C. (1982). f max. Bulletin of the Seismological Society of America, 72(6A), 1867-1879.

Hanks, T. C., \& McGuire, R. K. (1981). The character of high-frequency strong ground motion. Bulletin of the Seismological Society of America, 71(6), 2071-2095.

Harris, R. A., Barall, M., Archuleta, R. J., Dunham, E. M., Aagaard, B., Ampuero, J.-P., et al. (2009). The SCEC/USGS dynamic earthquake rupture code verification exercise. Seismological Research Letters, 80(1), 119-126. https://doi.org/10.1785/gssrl.80.1.119

Ida, Y. (1972). Cohesive force across tip of a longitudinal-shear crack and griffiths specific surface-energy. Journal of Geophysical Research, 77, 3796-3805. https://doi.org/10.1029/JB077i020p03796 
Kanamori, H., \& Brodsky, E. E. (2004). The physics of earthquakes. Reports on Progress in Physics, 67(8), 1429. https://doi.org/10.1063/1. 1387590

Kaneko, Y., Lapusta, N., \& Ampuero, J. P. (2008). Spectral element modeling of spontaneous earthquake rupture on rate and state faults: Effect of velocity-strengthening friction at shallow depths. Journal of Geophysical Research, 113, B09317. https://doi.org/10.1029/ 2007JB005553

Klinger, Y., Okubo, K., Vallage, A., Champenois, J., Delorme, A., Rougier, E., et al. (2018). Earthquake damage patterns resolve complex rupture processes. Geophysical Research Letters, 45, 10,279-10,287. https://doi.org/10.1029/2018GL078842

Knight, E. E., Rougier, E., \& Lei, Z. (2015). Hybrid optimization software suite (HOSS)—Educational version LA-UR-15-27013. United States: Los Alamos National Laboratory.

Koller, M. G., Bonnet, M., \& Madariaga, R. (1992). Modelling of dynamical crack propagation using time-domain boundary integral equations. Wave Motion, 16(4), 339-366. https://doi.org/10.1016/0165-2125(92)90022-T

Kostrov, B. V. (1974). Seismic moment and energy of earthquakes, and seismic flow of rock. Izvestiya, Academy of Sciences, USSR. Physics of the Solid Earth, 1, 23-44.

Lisjak, A., Grasselli, G., \& Vietor, T. (2014). Continuum-discontinuum analysis of failure mechanisms around unsupported circular excavations in anisotropic clay shales. International Journal of Rock Mechanics and Mining Sciences, 65, 96-115. https://doi.org/10.1016/j. ijrmms.2013.10.006

Ma, S., \& Andrews, D. (2010). Inelastic off-fault response and three-dimensional dynamics of earthquake rupture on a strike-slip fault. Journal of Geophysical Research, 115, B04304. https://doi.org/10.1029/2009JB006382

Madariaga, R. (1977). High-frequency radiation from crack (stress drop) models of earthquake faulting. Geophysical Journal of the Royal Astronomical Society, 51, 625-651. https://doi.org/10.1111/j.1365-246X.1977.tb04211.x

Manighetti, I., King, G., \& Sammis, C. G. (2004). The role of off-fault damage in the evolution of normal faults. Earth and Planetary Science Letters, 217(3), 399-408. https://doi.org/10.1016/S0012-821X(03)00601-0

Marty, S., Passelègue, F. X., Aubry, J., Bhat, H. S., Schubnel, A., \& Madariaga, R. (2019). Origin of high-frequency radiation during laboratory earthquakes. Geophysical Research Letters, 46, 3755-3763. https://doi.org/10.1029/2018GL080519

Mitchell, T. M., \& Faulkner, D. R. (2009). The nature and origin of off-fault damage surrounding strike-slip fault zones with a wide range of displacements: A field study from the Atacama fault system, northern Chile. Journal of Structural Geology, 31(8), 802-816. https:// doi.org/10.1016/j.jsg.2009.05.002

Mitchell, T. M., \& Faulkner, D. R. (2012). Towards quantifying the matrix permeability of fault damage zones in low porosity rocks. Earth and Planetary Science Letters, 339-340, 24-31. https://doi.org/10.1016/j.epsl.2012.05.014

Munjiza, A. A. (2004). The combined finite-discrete element method. Chichester: John Wiley \& Sons. https://doi.org/10.1002/0470020180

Munjiza, A. A., Knight, E. E., \& Rougier, E. (2011). Computational mechanics of discontinua. Chichester, West Sussex: John Wiley \& Sons. https://doi.org/10.1002/9781119971160

Munjiza, A., Knight, E. E., \& Rougier, E. (2015). Large strain finite element method: A practical course. Chichester, West Sussex: John Wiley \& Sons.

Ngo, D., Huang, Y., Rosakis, A. J., Griffith, W. A., \& Pollard, D. D. (2012). Off-fault tensile cracks: A link between geological fault observations, lab experiments, and dynamic rupture models. Journal of Geophysical Research, 117, B01307. https://doi.org/10.1029/ 2011JB008577

Nur, A., \& Simmons, G. (1969). The effect of saturation on velocity in low porosity rocks. Earth and Planetary Science Letters, 7(2), 183-193. https://doi.org/10.1016/0012-821X(69)90035-1

Ohnaka, M., Kuwahara, Y., \& Yamamoto, K. (1987). Constitutive relations between dynamic physical parameters near a tip of the propagating slip zone during stick-slip shear failure. Tectonophysics, 144(1-3), 109-125. https://doi.org/10.1016/0040-1951(87)90011-4

Okubo, K. (2018). Dynamic earthquake ruptures on multiscale fault and fracture networks. USA, Institut de Physique du Globe de Paris. https://hal.archives-ouvertes.fr/tel-02004905

Palmer, A. C., \& Rice, J. R. (1973). Growth of slip surfaces in progressive failure of over-consolidated clay. Proceedings of the Royal Society of London, Series A, 332, 527-548. https://doi.org/10.1098/rspa.1973.0040

Passelègue, F. X., Schubnel, A., Nielsen, S., Bhat, H. S., Deldicque, D., \& Madariaga, R. (2016). Dynamic rupture processes inferred from laboratory microearthquakes. Journal of Geophysical Research: Solid Earth, 121, 4343-4365,. https://doi.org/10.1002/2015JB012694

Poliakov, A. N. B., Dmowska, R., \& Rice, J. R. (2002). Dynamic shear rupture interactions with fault bends and off-axis secondary faulting. Journal of Geophysical Research, 107(B11), 2295. https://doi.org/10.1029/2001JB000572

Rice, J. R. (1980). The mechanics of earthquake rupture. In A. M. Dziewonski, \& E. Boschi (Eds.), Physics of the Earth's interior (Proc. Intn'l. School of Physics 'Enrico Fermi', Course 78, 1979) (pp. 555-649). Amsterdam: Italian Physical Society and North-Holland Publishing Co.

Rice, J. R., Sammis, C. G., \& Parsons, R. (2005). Off-fault secondary failure induced by a dynamic slip pulse. Bulletin of the Seismological Society of America, 95(1), 109-134. https://doi.org/10.1785/0120030166

Rivera, L., \& Kanamori, H. (2005). Representations of the radiated energy in earthquakes. Geophysical Journal International, 162(1), 148-155. https://doi.org/10.1111/j.1365-246X.2005.02648.x

Rojas, O., Day, S., Castillo, J., \& Dalguer, L. A. (2008). Modelling of rupture propagation using high-order mimetic finite differences. Geophysical Journal International, 172(2), 631-650. https://doi.org/10.1111/j.1365-246X.2007.03651.X

Rougier, E., Knight, E. E., Munjiza, A., Sussman, A. J., Broome, S. T., Swift, R. P., \& Bradley, C. R. (2011). The combined finite-discrete element method applied to the study of rock fracturing behavior in 3D, 45th US Rock Mechanics/Geomechanics Symposium. San Francisco, California: American Rock Mechanics Association.

Savage, H. M., \& Brodsky, E. E. (2011). Collateral damage: Evolution with displacement of fracture distribution and secondary fault strands in fault damage zones. Journal of Geophysical Research, 116, B03405. https://doi.org/10.1029/2010JB007665

Shi, Z., Ben-Zion, Y., \& Needleman, A. (2008). Properties of dynamic rupture and energy partition in a solid with a frictional interface. Journal of the Mechanics and Physics of Solids, 56(1), 5-24. https://doi.org/10.1016/j.jmps.2007.04.006

Shipton, Z. K., \& Cowie, P. A. (2001). Damage zone and slip-surface evolution over $\mu \mathrm{m}$ to km scales in high-porosity navajo sandstone, utah. Journal of Structural Geology, 23(12), 1825-1844. https://doi.org/10.1016/S0191-8141(01)00035-9

Sibson, R. H. (1977). Fault rocks and fault mechanisms. Journal of the Geological Society (London, United Kingdom), 133(3), 191-213. https://doi.org/10.1144/gsjgs.133.3.0191

Sowers, J. M., Unruh, J. R., Lettis, W. R., \& Rubin, T. D. (1994). Relationship of the Kickapoo fault to the Johnson Valley and Homestead Valley faults, San Bernardino county, California. Bulletin of the Seismological Society of America, 84(3), 528-536.

Templeton, E. L., \& Rice, J. R. (2008). Off-fault plasticity and earthquake rupture dynamics: 1. Dry materials or neglect of fluid pressure changes. Journal of Geophysical Research, 113, B09306. https://doi.org/10.1029/2007JB005529 
Thomas, M. Y., \& Bhat, H. S. (2018). Dynamic evolution of off-fault medium during an earthquake: A micromechanics based model. Geophysical Journal International, 214(2), 1267-1280. https://doi.org/10.1093/gji/ggy129

Viesca, R. C., \& Garagash, D. I. (2015). Ubiquitous weakening of faults due to thermal pressurization. Nature Geoscience, 8(11), 875-879. https://doi.org/10.1038/ngeo2554

Viesca, R., Rice, J., \& Dunham, E. M. (2009). Plastic deformation at a propagating rupture front: Its coupling to fault pore pressure and influence on the seismic moment tensor, Paper presented at Southern California Earthquake Center Annual Meeting. CA: Palm Springs.

Viesca, R. C., Templeton, E. L., \& Rice, J. R. (2008). Off-fault plasticity and earthquake rupture dynamics: 2. Case of saturated off-fault materials. Journal of Geophysical Research, 113, B09307. https://doi.org/10.1029/2007JB005530

Wallace, R. E., \& Morris, H. T. (1986). Characteristics of faults and shear zones in deep mines. Pure and Applied Geophysics, 124(1), 107-125. https://doi.org/10.1007/BF00875721

Xia, K. W., Rosakis, A. J., \& Kanamori, H. (2004). Laboratory earthquakes: The sub-rayleigh-to-supershear rupture transition. Science, 303, 1859-1861. https://doi.org/10.1007/s10704-006-0030-6

Xu, S., Ben-Zion, Y., \& Ampuero, J.-P. (2012). Properties of inelastic yielding zones generated by in-plane dynamic ruptures-I. Model description and basic results. Geophysical Journal International, 191(3), 1325-1342. https://doi.org/10.1111/j.1365-246X.2012.05679.x

Yamashita, T. (2000). Generation of microcracks by dynamic shear rupture and its effects on rupture growth and elastic wave radiation. Geophysical Journal International, 143(2), 395-406. https://doi.org/10.1046/j.1365-246X.2000.01238.x 